\title{
Die Reform der direkten Demokratie in Berlin 2006 (Teil I)
}

\section{Otmar Jung}

Formell geht es in der folgenden Studie nur um die Verbesserung des Regelwerks der Volksgesetzgebung in einem Bundesland, doch kommen bei der Analyse jener Reform alle grundsätzlichen Probleme zum Vorschein, mit denen die direkte Demokratie in Deutschland auf Landesebene konfrontiert ist. Teil I behandelt den Reformprozeß 2006. In Teil II folgt die inhaltliche Beurteilung der Reform.

\section{Einleitung}

Vom Kriegsende bis zur Verfassungsgebung 1950 befand sich (West-)Berlin, was direkte Demokratie angeht ${ }^{1}$, durchaus im gemeindeutschen Takt ${ }^{2}$. Eine Sonderentwicklung begann, als Senat und Abgeordnetenhaus es fast ein Vierteljahrhundert lang unterließen, das einschlägige Regelwerk der Verfassung durch ein Ausführungsgesetz handhabbar zu machen, und schließlich 1974 die Volksgesetzgebung aus der Verfassung strichen. Dies war ein in Deutschland bisher einmaliger Vorgang, den man juristisch als „Staatsstreich“33 und aus der Sicht des demokratischen politischen Systems als „Amputation“ qualifiziert hat ${ }^{4}$, dessen Hintergründe in den Statusquerelen der geteilten Frontstadt des Kalten Krieges

$1 \mathrm{Zu}$ den bisherigen Erfahrungen mit direkter Demokratie in Berlin vgl. allgemein C. Pestalozza, Auf gutem Weg: Direkte Demokratie in Berlin, in: JdD 1 (2009), S. 295316 (296-304); speziell F. Meerkamp, Die Quorenfrage im Volksgesetzgebungsverfahren. Bedeutung und Entwicklung, Wiesbaden 2011 (Bürgergesellschaft und Demokratie Bd. 36), S. 108-120.

2 Vgl. O. Jung, Grundgesetz und Volksentscheid. Gründe und Reichweite der Entscheidungen des Parlamentarischen Rats gegen Formen direkter Demokratie, Opladen 1994, S. 131-139.

3 Vgl. K.A. Schachtschneider, Gesetzgebung und Verfassungsänderung durch das Volk in Berlin, in: JR 1975, S. 221-224 (223 Fn. 19).

4 Vgl. O. Jung, Weniger Demokratie wagen? - Seltsames aus der Berliner Verfassungsrevision -, in: JR 1996, S. 1-10 (2). 
lagen $^{5}$ und der im Parlament mit Argumenten begründet wurde, die zeigen, daß politische Kurzsichtigkeit nicht nur beim deswegen oft gescholtenen Volk, sondern ebenso bei seinen Vertretern zu finden ist.

Nach der Wiedervereinigung der Stadt wurde der Druck vor allem aus dem Ostteil überstark, in die neue Verfassung von 1995 Volksbegehren und Volksentscheid wieder aufzunehmen. Dies geschah, doch der Widerstand der hier zum Nachgeben gezwungenen politischen Elite des alten West-Berlin setzte sich auf tieferer Ebene fort. Die Verfassungsregelung zur direkten Demokratie atmete spürbar „das Mißbehagen ihrer Autoren“6, und die Ausführungsgesetzgebung verschärfte die Bedingungen für das „ungeliebte Modell“ direkte Demokratie noch $^{7}$.

Die neue Volksbegehrenshürde von zehn Prozent mochte moderat wirken, aber die alte, d. h. schon in der Verfassung von 1950 enthaltene, alternative „Berliner Doppelhürde“ - bei 50 Prozent Beteiligung genügte die einfache Mehrheit, bei geringerer Beteiligung war die Zustimmung von 33 Prozent der Stimmberechtigten erforderlich - erschien unüberwindbar. Auf fielen die weiten Tabubereiche, darunter eine Spezialität: Auf Verlangen der stärksten Partei, der CDU, tabuisierte Berlin als einziges Bundesland auch die Verfassung, schloß also die verfassungsändernde Volksgesetzgebung aus ${ }^{8}$.

Die Praxis der direkten Demokratie begann gar nicht schlecht. Das Referendum 1995, mit dem die neue Verfassung angenommen wurde ${ }^{9}$ - der erste Volksentscheid in Berlin auf Landesebene überhaupt -, und die Doppel-Volksabstimmung 1996 über die Länderfusion Berlin-Brandenburg, bei der das Projekt in Berlin gebilligt wurde ${ }^{10}$, liefen ordnungsgemäß ab. Aber dies war direkte Demokratie „von oben“. Die ersten Versuche der Volksgesetzgebung hingegen - also

5 Siehe die offiziöse Begründung für die „Eliminierung“ bei R.-P. Magen in: G. Pfennig/ M.J. Neumann (Hrsg.), Verfassung von Berlin. Kommentar, 3. Aufl. Berlin 2000, Art. 2 Rn. 4, Art. 61 Rn. 6.

6 C. Pestalozza, Die überarbeitete Verfassung von Berlin. Integrationsbeitrag und Fusionsmitgift, in: LKV 5 (1995), S. 344-353 (350).

7 Vgl. A. Ziekow, Direkte Demokratie in Berlin. Entwicklung und Ausgestaltung eines ungeliebten Modells, in: LKV 9 (1999), S. 89-94.

8 Vgl. zum Entscheidungsprozeß O. Jung, Abschluß und Bilanz der jüngsten plebiszitären Entwicklung in Deutschland auf Landesebene, in: JöR 48 (2000), S. 39-85 (60-65). Diese Tabuisierung war noch stärker als im Saarland, wo Volksbegehren zur Änderung der Verfassung möglich sind, über die dann allerdings kein Volksentscheid stattfindet (Art. 100 Abs. 4 SaarlVerf.).

9 Vgl. O. Jung, Volksentscheide in der Bundesrepublik. Eine aktuelle Übersicht, in: Blätter für deutsche und internationale Politik 41 (1996), S. 567-576 (574 ff.).

10 Vgl. O. Jung, Die Volksabstimmungen über die Länderfusion Berlin - Brandenburg: Was hat sich bewährt - wer ist gescheitert? in: ZParl 28 (1997), S. 13-20. 
direkte Demokratie „von unten“ - schlugen allesamt fehl ${ }^{11}$ : Die Volksinitiative gegen den Bau des Transrapids Berlin-Hamburg zeigte vor allem die Mängel des eingesetzten direktdemokratischen Instruments; das Volksbegehren „Mehr Demokratie in Berlin" scheiterte vor dem Verfassungsgerichtshof, der das Verfassungstabu weit auslegte, und das Volksbegehren „Schluß mit der Rechtschreibreform!"“ hatte keinen Erfolg wegen der Überforderung der Amateur-Initiatoren und der schwierigen Bedingungen in der Millionenstadt, aber auch wegen des Regelwerks, von dem ein Fachmann schon während der Verfassungsgebung mit Blick auf die Verfahrenshürden den Eindruck gewonnen hatte, ,es sei den Überarbeitern um die Verhinderung von [Volks-]Begehren gegangen“"12.

Nach der Aufdeckung des Berliner Bankenskandals ${ }^{13} 2001$ schied die SPD aus der Großen Koalition aus, verbündete sich mit den Grünen und stürzte, unterstützt von der PDS, den Regierenden Bürgermeister E. Diepgen (CDU). Es folgten ein rot-grüner Minderheitssenat und Neuwahlen, welche die Erschütterung des politischen Gefüges der Stadt ausdrückten. Die CDU erlebte einen „Höllensturz“ (H. Prantl) und verlor mehr als die Hälfte ihrer Mandate ${ }^{14}$. Die Regierungsmacht übernahm 2002 ein rot-roter Senat. In ihrem Koalitionsvertrag vereinbarten die Partner, daß sie ,die demokratischen Mitwirkungsrechte der Bürgerinnen und Bürger verbessern“ wollten. Auf Bezirksebene war dazu der Handlungsbedarf besonders eklatant. Berlin hatte zwar 1978 das Bürgerbegehren in den Bezirken eingeführt, das Verfahren aber imperfekt gestaltet. Den Siegeszug des kommunalen Bürgerentscheids in den anderen Bundesländern in den 1990er Jahren ${ }^{15}$ ließ Berlin ungerührt, möchte man sagen, an sich vorbeiziehen und stand nun, was die kommunale sachpolitische Direktpartizipation angeht, als

11 Vgl. O. Jung, Dreimal Fehlschlag. Die schwierigen Anfänge der direkten Demokratie in Berlin, in: ZParl 32 (2001), S. 33-57; J. Rux, Direkte Demokratie in Deutschland. Rechtsgrundlagen und Rechtswirklichkeit der unmittelbaren Demokratie in der Bundesrepublik Deutschland und ihren Ländern, Baden-Baden 2008, S. 780 ff., 787 f.

12 Vgl. Pestalozza, Verfassung (Fn. 6), S. 351 (Hervorhebung i.O.).

13 Als ein Untersuchungsausschuß des Landesparlaments 2006 seinen Abschlußbericht vorlegte, nannte der Ausschußvorsitzende Frank Zimmermann (SPD) als Hauptgründe für den Bankenskandal: „krasses Mißmanagement, politisches Versagen, Größenwahn, kriminelle Energie und ein System der Verschleierung“ (Der Tagesspiegel [Tsp.] Nr. 19209 v. 3.6.2006).

14 Vgl. O. Niedermayer/R. Stöss, Die Wahl zum Berliner Abgeordnetenhaus vom 21. Oktober 2001: Regierungswechsel nach vorgezogenen Neuwahlen, in: ZParl 33 (2002), S. 244-261 (250 f., 258).

15 Vgl. O. Jung, Siegeszug direktdemokratischer Institutionen als Ergänzung des repräsentativen Systems? Erfahrungen der 90er Jahre, in: H.H. v. Arnim (Hrsg.), Demokratie vor neuen Herausforderungen. Vorträge und Diskussionsbeiträge auf dem 1. Speyerer Demokratie-Forum vom 29. bis 31. Oktober 1997 an der Deutschen Hochschule für Verwaltungswissenschaften Speyer, Berlin 1999 (Schriftenreihe der Hochschule Speyer Bd. 130), S. 103-137 (107 f., 115, 127-137). 
Schlußlicht da. Künftig sollten also „Bürgerentscheide auf der Basis von Bürgerbegehren ... ermöglicht" werden ${ }^{16}$, und in der Tat setzten Senat und Abgeordnetenhaus dies 2005 in einer ersten großen Direkte-Demokratie-Reform um ${ }^{17}$. Der Fachverband Mehr Demokratie e.V. bemerkte anerkennend: „Berlin bekommt das beste Bürgerbeteiligungsgesetz, das je von einem deutschen Landesparlament verabschiedet wurde. “18 Hier zeichnete sich eine Wende der politischen Klasse Berlins ab.

Daß im Herbst dieses Jahres ein weiteres Volksbegehren („Schluß mit dem Berliner Bankenskandal") vor dem Verfassungsgerichtshof scheiterte, der nun auch das Finanztabu weit auslegte ${ }^{19}$, kehrte die politische Großströmung nicht mehr um.

16 Die einschlägigen Passagen der Koalitionsvereinbarung vom 16.1.2002 sind abgedruckt bei C. Posselt, Direkte Demokratie in Berlin, in: A. Kost (Hrsg.), Direkte Demokratie in den deutschen Ländern. Eine Einführung, Wiesbaden 2005, S. 60-74 (62 f. Fn. 4).

17 Fünftes Gesetz zur Änderung der Verfassung von Berlin v. 28.6.2005, GVB1. S. 346. Die erste direktdemokratische Phase auf der Bezirksebene 1978-2005 wird gern übersehen und die Einführung der kommunalen Direktdemokratie in Berlin insgesamt erst auf 2005 datiert, so T. Schiller, Direkte Demokratie auf Bundesländer- und Kommunalebene, in: M. Freitag/U. Wagschal (Hrsg.), Direkte Demokratie. Bestandsaufnahmen und Wirkungen im internationalen Vergleich, Berlin 2007 (Policy-Forschung und Vergleichende Regierungslehre Bd. 3), S. 115-150 (128); P. Neumann, Sachunmittelbare Demokratie im Bundes- und Landesverfassungsrecht unter besonderer Berücksichtigung der neuen Länder, Baden-Baden 2009, S. 125 mit nicht überzeugender Begründung; C. Eder, Direkte Demokratie in den deutschen Kommunen. Regelungen zu Bürgerbegehren und Bürgerentscheiden im Vergleich, in: M. Freitag/A. Vatter (Hrsg.), Vergleichende subnationale Analysen für Deutschland. Institutionen, Staatstätigkeiten und politischen Kulturen, Berlin 2010 (Policy-Forschung und Vergleichende Regierungslehre Bd. 7), S. 65-95 (67); T. Schiller, Local direct democracy in Germany - varieties in a federal state, in: ders. (Hrsg.), Local Direct Democracy in Europe, Wiesbaden 2011 (Direct Democracy in Modern Europe), S. 54-74 (56). - Zu den Erfahrungen mit dem Bürgerbegehren 1978-2005 vgl. M. Efler/C. Posselt, Direkte Demokratie in Berlin, in: H.K. Heußner/O. Jung (Hrsg.), Mehr direkte Demokratie wagen. Volksentscheid und Bürgerentscheid: Geschichte - Praxis - Vorschläge, 2. Aufl. München 2009, S. 285-304 (294 ff.).

18 C. Posselt, Der weiße Fleck ist weg! Bürgerbegehren und Bürgerentscheide in Berlins Bezirken eingeführt, in: mehr demokratie. Zeitschrift für Direkte Demokratie 16 (2005), H. 3 (Nr. 67), S. 24. Skeptisch J. Platter, Der Bürgerentscheid in den Berliner Bezirken Erweiterung demokratischer Teilhaberechte oder basisdemokratische Attrappe? In: LKV 16 (2006), S. 295-300. - Zur Praxis siehe Mehr Demokratie e. V. [M. Efler/S. Gröger/ D. Urech], Zweiter Berliner Demokratiebericht, Berlin 2008, S. 20-35, 45-56.

19 Verfassungsgerichtshof des Landes Berlin, Urt. v. 22.11.2005, LVerfGE 16, 41. 


\section{Der Reformprozeß 2006}

Der Koalitionsvertrag von 2002 sah auch eine Verbesserung der direkten Demokratie auf Landesebene vor ${ }^{20}$, doch war es mitnichten so, daß der hier zu betrachtende Reformprozeß 2006 einfach als Exekution jener Vereinbarung begonnen hätte. Es war ein offenen Geheimnis, daß es bei den Sozialdemokraten „erhebliche Widerstände“" gegen das Projekt gab ${ }^{21}$, und die Parteiführung hatte deshalb bislang die Marschroute ausgegeben, mit der Reform der Volksgesetzgebung bis zur nächsten Legislaturperiode zu warten ${ }^{22}$. Der politische Impetus kam ganz woanders her, und die Reform der direkten Demokratie in Berlin gelang aufgrund eines klassischen politischen „Deals ${ }^{\text {“23 }}$ der Parteien im Abgeordnetenhaus.

\subsection{Der Impetus der politischen Elite}

Alles begann mit dem Entschluß der Landesspitze der SPD um den Regierungschef $K$. Wowereit, die Realisierung eines lange gehegten Wunsches nun ernsthaft anzugehen und ein Strukturmerkmal der Berliner Verfassung zu ändern: Man wollte das stadtstaatliche Senatsprinzip (vor allem bei der Regierungsbildung) durch das vom Bund und den Flächenländern her bekannte Kanzlerprinzip ersetzen. Aus dem „Regierenden Bürgermeister“ sollte sozusagen ein „Ministerpräsident" werden.

Diese Reform als solche ist nicht Gegenstand der Untersuchung. Deshalb hier nur vier Bemerkungen: Erstens dürfte dabei ein Trauma der SPD mitgespielt haben, die bei der bisher gebotenen Wahl jedes einzelnen Senators durch das Abgeordnetenhaus (Art. 56 Abs. 2 VvB a.F.) wegen Stimmverweigerung aus den eigenen Reihen bzw. denen des Koalitionspartners - die sogenannten U-Boote 1981 einen ganzen Senat (unter D. Stobbe) und nach einem viermonatigen Übergang (unter H.-J. Vogel) auch noch (für acht Jahre) die Macht verloren und zuletzt 2002 bei der Wahl ihres Landesvorsitzenden P. Strieder zum Stadtentwicklungssenator erhebliches Ungemach erlebt hatte. Zweitens: Während Hamburg

20 Vgl. Fn. 16. Die einschlägigen Passagen lauteten: „Direkte Demokratie auf Landesebene soll durch eine Vereinfachung der formalen Voraussetzungen für Volksinitiative, Volksbegehren und Volksentscheid (vereinfachte Sammlungsbedingungen, angemessene Fristen) erleichtert werden. Die Koalitionsparteien streben dabei auch eine Absenkung der Quoren an. Zusätzlich prüfen wir, ob die Möglichkeiten für Volksbegehren erweitert werden können.“

21 Vgl. Mehr Demokratie Berlin, Pressemitteilung (PM) Nr. 1/06 v. 12.1.2006.

22 Vgl. Abg. Klaus Lederer (Linkspartei.PDS), Abgeordnetenhaus (AH) 4.5.2006, S. 7342 A; Abg. Volker Ratzmann (Bündnis 90/Die Grünen), a.a.O., S. 7345 A.

23 So der Ausdruck bei C. Posselt, Berlin sagt Ja zur Volksabstimmung, in: mehr demokratie 17 (2006), H. 3 (Nr. 71), S. 34. 
seine Verfassung 1996 behutsam modernisiert hatte dergestalt, daß für die Regierungsbildung nunmehr zwei parlamentarische Mitwirkungsakte erforderlich, aber auch ausreichend sind - zunächst die Wahl des Ersten Bürgermeisters und danach die Bestätigung aller weiteren Mitglieder des Senats en $b \operatorname{loc}^{24}-$, wollte die politische Elite Berlins jetzt „durchreformieren“. Drittens bedeutete eine solche radikale Angleichung einen Verlust an bundesstaatlicher Vielfalt. Viertens zeigte gleich die Premiere des neuen Systems, als der Kandidat Wowereit im ersten Anlauf nicht die erforderliche Mehrheit erhielt, daß die Reform an der Oberfläche geblieben war. Die zugrundeliegende Spannung zwischen dem durch das Abstimmungsgeheimnis geschützten freien Mandat der Abgeordneten und den Zwängen des parlamentarischen Regierungssystems, in dem die Mehrheitsfraktionen die Regierung stellen und stützen müssen, blieb unaufhebbar. Erreicht wurde lediglich, daß diese Spannung nur einmal und nicht, wie beim StobbeSenat 1981, gleich viermal ausgehalten werden mußte - um den Preis, daß, wo es früher, z. B. 2002, um einen demütigenden Denkzettel ging, jetzt gleich eine Regierungskrise drohte.

Für dieses Vorhaben eine verfassungsändernde Mehrheit zu organisieren war deshalb schwierig, weil sachpolitisch eine Asymmetrie bestand: Die Regierungspartei SPD sowie die große Oppositionspartei CDU und die kleine oppositionelle FDP waren für den angestrebten Systemwechsel, die Koalitionspartnerin Linkspartei.PDS sowie die kleine Oppositionsfraktion der Grünen waren dagegen. Wenn also die SPD die Ad-hoc-Koalition wieder zusammenfügen wollte, die bereits 2005 das 5. und 6. Gesetz zur Änderung der Verfassung von Berlin - die Senkung des aktiven Wahlalters für die Bezirksebene auf 16 Jahre und die Einführung des Bürgerentscheids in den Bezirken - getragen hatte, mußte sie diese beiden Fraktionen umstimmen. In dieser Situation traf die SPD-Führung die strategische Entscheidung, der Linkspartei.PDS und den Grünen eine Reform der direkten Demokratie auf Landesebene als Köder anzubieten. Der Übergang zum Kanzlerprinzip und die Verbesserung der Volksrechte sollten zusammen verabschiedet werden (Zweier-Paket). Dieser Köder hatte einen beträchtlichen Reiz:

- Daß die SPD-Führung ihren bisherigen Kurs des Abwartens aufgab und sich, gegen die Widerstände in den eigenen Reihen, bereit erklärte, diese Reform jetzt doch noch - wenige Monate vor der nächsten Wahl zum Abgeordnetenhaus, zugleich dem geeigneten Termin für die obligatorische Volksabstimmung zu dieser Frage ${ }^{25}$ - anzupacken, bedeutete durchaus ein gewichti-

24 Vgl. Art. 34 Abs. 1 und 2 S. 2 HmbVerf. und K. David, Verfassung der Freien und Hansestadt Hamburg. Kommentar, 2. Aufl. Stuttgart u.a. 2004, Art. 35 Rn. 1 f., 6-10.

25 Daß hier ein „Fenster der Gelegenheit“ offenstand, das sich so bald nicht wieder öffnen würde, war den politischen Profis im Abgeordnetenhaus von vornherein klar, vgl. Abg. Ratzmann (Bündnis 90/Die Grünen), AH 2.6.2005, S. 5840 B; Abg. J. Martins (Bündnis 
ges Entgegenkommen. Böse formuliert, erklärte sich die Partei bereit, die Kröte, die zu schlucken sie schon vor vier Jahren versprochen, deren Verzehr sie dann aber ,auf die lange Bank“ geschoben hatte, jetzt tatsächlich hinunterzuwürgen.

- Zugleich konnte die SPD gegenüber der Linkspartei.PDS und der Öffentlichkeit das Gesicht wahren, indem sie das Lockangebot als bloße Umsetzung der Abrede im Koalitionsvertrag ausgab.

- Während der Wechsel vom Senats- zum Kanzlerprinzip im Kern eine harte Alternative bedeutete, eignete sich die ,weiche“ Forderung nach einer Erleichterung der direkten Demokratie auf Landesebene - man denke nur an das geradezu skalierbare Vorhaben einer Absenkung der Quoren - ideal zum Austarieren von Kompromissen.

Dabei war die Position der Koalitionspartnerin Linkspartei.PDS in dieser Hinsicht gut konturiert. 16 Monate vor Abschluß jener Koalitionsvereinbarung hatte sie mit einem Antrag über „Mehr Demokratie in Berlin“ ihr verfassungspolitisches Profil festgelegt ${ }^{26}$. Dieses noch in der Opposition erarbeitete ReformRegelwerk für die direkte Demokratie in Berlin gibt sozusagen die Position der „PDS pur“ wieder, ohne die Abschleifungen durch Mit-Regierungszwänge. Fünf Jahre später - aber noch vor Beginn des letzten, schließlich zum Erfolg führenden Reformanlaufs - hielt die Fraktion immer noch die damaligen Vorstellungen für maßgeblich ${ }^{27}$.

Bitter war nach jener Entscheidung der SPD-Führung die Lage der CDU. Wieder wurde deutlich, welche strategische Niederlage sie 2001 bei den Neuwahlen nach dem Berliner Bankenskandal erlitten hatte, als sie ihre bis dahin behauptete Sperrminorität verlor ${ }^{28}$. Bei den beiden erwähnten demokratiepolitischen Reformen 2005 war der CDU die Tatsache, daß gegen ihren Willen die Spielregeln geändert werden konnten, demonstrativ vor Augen geführt worden.

- Wenn die CDU ablehnte - bitte. Die Ad-hoc-Zusammenarbeit des Vorjahres zwischen der Koalition und den beiden kleinen Oppositionsparteien würde auch 2006 funktionieren.

- Wenn die CDU „mitmachen“ wollte, würde man sie nicht zurückweisen sie konnte immerhin für die erwünschte politische Optik einer einmütigen

90/Die Grünen), AH 16.6.2005, S. 5950 A; Abg. Lederer (Linkspartei.PDS), AH 24.11.2005, S. 6550 A.

26 „Mehr direkte Demokratie in Berlin (I)“ mit Entwurf eines „Vierten Gesetzes zur Änderung der Verfassung von Berlin (VvB)“, Drs. 14/655 v. 14.9.2000.

27 Vgl. Abg. Lederer (Linkspartei.PDS), AH 24.11.2005, S. 6550 B (mit Verwechslung des Jahres: ,2001“ statt richtig ,2000“).

28 In der 14. Wahlperiode 1999-2001 war gegen die CDU mit 76 von 169 Mandaten keine Verfassungsänderung möglich. Nach den Neuwahlen 2001 verfügte sie nur noch über 35 von 141 Mandaten. 
Verfassungsänderung sorgen ${ }^{29}$. Aber daraus folgte nicht viel. Insbesondere brachte es die SPD nicht davon ab, die Linkspartei.PDS und die Grünen mit der Reform der direkten Demokratie als Köder zu locken. Mochte die CDU dies als Kröte empfinden - warum sollte es ihr besser ergehen als der Sozialdemokratie selbst?

In dieser Situation traf auch die CDU-Führung eine strategische Entscheidung. Anders als im Vorjahr, als sie in zwei Fällen sozusagen „mit wehenden Fahnen“ untergegangen war, erklärte sich die CDU nun bereit, die Reform der direkten Demokratie mitzutragen, um wenigstens etwas auf die konkrete Ausgestaltung dieser Konzession an die Linkspartei.PDS und die Grünen Einfluß nehmen zu können.

Nach diesem Drehbuch lief nun der Entscheidungsprozeß ab. Schon seit Herbst 2005 gab es „Verständigungsgespräche“30, seit Anfang des Jahres tagte eine interfraktionelle Arbeitsgruppe der Parteien des Abgeordnetenhauses ${ }^{31}$. Am 12. Januar warf Bürgermeister Wowereit in einem „tageszeitungs“-Interview den Köder der Direkte-Demokratie-Reform aus ${ }^{32}$ - setzte damit natürlich auch die Widerstrebenden in seiner eigenen Partei unter Druck -, und eine Woche später war schon das Zweier-Paket geschnürt ${ }^{33}$. Das Kalkül der SPD-Führung war aufgegangen. Ihre Koalitionspartnerin Linkspartei.PDS, für die das Kanzlerprinzip „eigentlich nicht in Betracht“ gekommen war, hatte auf den Köder angebissen. Zusammen mit einer Reform der direkten Demokratie war jene Straffung der

29 In der Tat waren von den bis dahin fünf Verfassungsänderungen der 15. Legislaturperiode drei konsensuell und nur zwei kontrovers erfolgt. Auch das hier behandelte 8. Gesetz zur Änderung der Verfassung von Berlin sowie das gleich darauf folgende 9. Änderungsgesetz sollten (im wesentlichen) einstimmig verabschiedet werden. Den politischen „Eigenwert" der Tatsache, daß alle Fraktionen mitmachten, betonte Abg. Lederer (Linkspartei.PDS), AH 4.5.2006, S. 7342 B.

30 Vgl. Abg. A. Ritzmann (FDP), AH 4.5.2006, S. 7346 D.

31 Ihr gehörten an C. Gaebler (SPD), F. Henkel (CDU), Lederer (Linkspartei.PDS), Ratzmann (Bündnis 90/Die Grünen) und Ritzmann (FDP). Es handelte sich um relativ junge Politiker (zur Jahreswende zwischen 33 und 45 Jahre alt) mit überwiegend sehr kurzer Mandatszeit (nur Gaebler gehörte dem Abgeordnetenhaus bereits seit 1995 an, alle anderen waren erst 2001 gewählt worden bzw. später nachgerückt), die gleichwohl in ihren Parteien und Fraktionen überwiegend gut verankert waren (Gaebler und Henkel als parlamentarische Geschäftsführer, Lederer als Landes- und Ritzmann als stellvertretender Fraktionsvorsitzender; der letztgenannte sollte allerdings im Frühjahr 2006 einen innerparteilichen Machtkampf verlieren und nicht wieder für das Abgeordnetenhaus kandidieren). Sie hatten alle studiert, aber nur zwei waren Juristen, dazu kam ein Politologe. Insgesamt handelte hier eine ganz andere politische Generation als jene, welche die Verfassung von 1995 erarbeitet hatte.

32 Vgl. „Kurz: Alles wird noch besser“, in: die tageszeitung (taz) Nr. 7869 v. 12.1.2006.

33 Vgl. U. Zawatka-Gerlach, Mehr Macht für den Regierenden und das Volk, in: Tsp. Nr. 19077 v. 18.1.2006; ders., Mehr Macht für den Regierungschef, in: Tsp. Nr. 19079 v. 20.1.2006. 
Regierungsorganisation für die Sozialisten „dann doch vorstellbar“34. Bei den Oppositionsparteien konnten die Grünen das Angebot der größeren Regierungspartei nicht ,ausschlagen“ und begaben sich „mit Schmerzen“ in den Verhandlungsprozeß („Uns war jedoch die direkte Demokratie so wichtig“) $)^{35}$. Der FDP fiel die Zustimmung leicht, weil sie sachpolitisch für beide Teile des Pakets war $^{36}$. Die CDU endlich zog mit, weil mit der Stärkung der Position des Regierenden Bürgermeisters für sie „ein seit langem gehegter Wunsch in Erfüllung“ ging $^{37}$ und weil sie in den Verhandlungen dafür sorgen konnte, daß man der Linkspartei nicht zu weit entgegenkam - was diese sehr wohl spürte ${ }^{38}$.

Demnächst brachten die Grünen eine dritte Forderung ein: den Ausbau der Kontrollrechte der einzelnen Abgeordneten bzw. des Parlaments insgesamt, soweit dies die Einsicht in Akten der Verwaltung und die Befragung von Vertretern des Landes Berlin in Unternehmen im Eigentum oder unter maßgeblichem Einfluß des Landes betraf ${ }^{39}$. Diese Forderung war unter den Fraktionen unstreitig; man empfand solche erweiterten Informationsrechte für Abgeordnete gewissermaßen als eine Kompensation an die parlamentarisch-repräsentative Demokratie für ihre Einbußen durch den Ausbau der Volksrechte, und dies zu Lasten eines Dritten: der Regierung bzw. Verwaltung. So wurde aus dem Zweier- ein Dreier-Paket $^{40}$. Mitte März hatten sich die Fraktionen fast schon auf den Endstand geeinigt, wenngleich die Detailarbeit noch bis zum Beginn der parlamentarischen Phase weiterging. Wie vortrefflich sich das Projekt der Reform der direkten Demokratie zum Austarieren des Gesamtpakets eignete, zeigt das Detail, daß

34 Abg. Lederer (Linkspartei.PDS), AH 4.5.2006, S. 7341 B.

35 Vgl. Abg. Ratzmann (Bündnis 90/Die Grünen), AH 4.5.2006, S. 7345 CD.

36 Vgl. Abg. Ritzmann (FDP), AH 4.5.2006, S. 7346 D.

37 Abg. Henkel (CDU), AH 4.5.2006, S. 7342 D.

38 Vgl. Abg. Lederer (Linkspartei.PDS), AH 4.5.2006, S. 7342 B; ferner Abg. Henkel (CDU), a.a.O., S. 7342 C, 7343 A. Der Abg. Ritzmann (FDP) brachte es auf den Punkt: „Dadurch, daß die Union an diesem Projekt teilgenommen hat ..., haben sich die Machtverhältnisse ... in der Arbeitsgruppe etwas verändert.“ (a.a.O., 7347 B). - Tief blicken läßt allerdings auch die (vermutlich zutreffende) Einschätzung der CDU, daß die Sozialdemokraten - Gaebler - ,teilweise deutlich strenger und rigider sein wollten als wir“, so Abg. Henkel (CDU), a.a.O., S. 7343 B.

39 Vgl. U. Zawatka-Gerlach, Mehr Rechte für alle, in: Tsp. Nr. 19120 v. 2.3.2006.

40 Diese politische Struktur des Kompromiß-Pakets wurde in dem nachfolgenden Allparteien-Antrag mit dem Entwurf eines Achten Gesetzes zur Änderung der Verfassung von Berlin nicht recht deutlich, der sich an der Verfassungssystematik orientierte und deshalb mit den neuen parlamentarischen Kontrollrechten begann (zum Abschnitt III. Die Volksvertretung), dann den Übergang vom Senats- zum Kanzlerprinzip regelte (zum Abschnitt IV. Die Regierung) und schließlich die Reform der direkten Demokratie brachte (zum Abschnitt V. Die Gesetzgebung). 
man bei der Qualifizierungshürde für ein Volksbegehren schließlich um halbe Prozentpunkte verhandelte ${ }^{41}$.

Das Abgeordnetenhaus verabschiedete die so entstandene All-Parteien-Vorlage ${ }^{42}$ so rasch wie eigenartig. In der I. Lesung am 4. Mai ${ }^{43}$ sprach je ein Redner für jede Fraktion - es waren die Unterhändler der interfraktionellen Arbeitsgruppe -, der sich im allgemeinen darauf beschränkte, den Weg seiner politischen Gruppierung zu dieser All-Parteien-Vorlage zu skizzieren und den Standort des gefundenen Kompromisses im eigenen politischen Weltbild zu markieren. Inhalte spielten keine Rolle. Kontroversen fanden nicht statt. Was an den bisherigen Regelungen im einzelnen unbefriedigend war, welche Konzeption von direkter Demokratie die Verantwortlichen für den Kompromiß leitete, warum man das Verfahren an dieser Stelle und nicht an jener so weit oder eben nicht so weit erleichtert hatte, wie die Berliner Regelung sich im gemeindeutschen Vergleich oder gar nach den Maßstäben alter demokratischer Länder mit ausgebauten Volksrechten ausnahm - dazu gab es im Parlament keine Auskunft, die über Formeln hinausgegangen wäre. Der Rechtsausschuß beschränkte sich im wesentlichen darauf, die Vorlage noch einmal rechtstechnisch durchzuarbeiten ${ }^{44}$. In der II. Lesung war - wie üblich ${ }^{45}$ - erst gar keine Aussprache vorgesehen ${ }^{46}$, sondern die Reform wurde umgehend einmütig (bei einer Gegenstimme) beschlossen ${ }^{47}$. Wenn nicht ein einzelner FDP-Abgeordneter aus diesem geradezu überwältigenden (in jedem Sinne) Konsens ausgeschert wäre und offen seine Bedenken zu-

41 Vgl. Mehr direkte Demokratie geplant, in: Tsp. Nr. 19134 v. 16.3.2006: 7,5 Prozent; U. Zawatka-Gerlach, Regierender wird mächtig wie ein Ministerpräsident, in: Tsp. Nr. 19175 v. 28.4.2006: 7 Prozent.

42 Drs. $15 / 5038$ v. 26.4.2006, Entwurf eines Achten Gesetzes zur Änderung der Verfassung von Berlin.

43 AH, 4.5.2006, S. $7340 \mathrm{C}-7348$ A.

44 AH Ausschuß für Verfassungs- und Rechtsangelegenheiten, Immunität und Geschäftsordnung, Sitzung v. 11.5.2006, Beschlußprotokoll - Recht - 15/70, S. 1-3, http://www. parlament-berlin.de/ados/Recht/protokoll/r15-070-bp.pdf, Inhaltsprotokoll - Recht 15/70, S. 2 f., http://www.parlament-berlin.de/ados/Recht/protokoll/r15-070-ip.pdf.

45 Es wird durchaus nicht übersehen, daß das Abgeordnetenhaus bei allen sieben Verfassungsänderungen der 15. Legislaturperiode so verfuhr. Indes ist zwischen mehr ,technischen" und sozusagen hochpolitischen Verfassungsänderungen zu unterscheiden, und bei letzteren erscheint der Verzicht auf Kommunikation - Vermittlung und Werbung für das Projekt - nicht verständlich.

46 Ein solches Vorgehen war nach $\S 33$ Abs. 3 der Geschäftsordnung (GO) des Abgeordnetenhauses von Berlin i.d.F. der Bek. v. 28.4.2005 (GVB1. S. 262, 463), geändert am 27.10.2005 (GVB1. S. 707) - zulässig; einen politischen Fehler bedeutete es gleichwohl, und dieser verlor auch nicht dadurch seinen Charakter, daß er immer wieder begangen wurde.

47 AH 18.5.2006, S. 7429 D - 7430 A. Das Achte Gesetz zur Änderung der Verfassung von Berlin wurde am 25.5.2006 ausgefertigt und im GVBl. S. 446 (Nr. 19 v. 3.6.2006) verkündet. 
mindest nachträglich zu Protokoll gegeben hätte ${ }^{48}$, wäre diese Verfassungsänderung völlig intransparent verlaufen.

In dieser Verfahrensweise drückte sich ein bestimmtes Verständnis von parlamentarischer Demokratie aus, das sich im Abgeordnetenhaus - durchaus umstritten, gerade beim Thema Reform der direkten Demokratie - gegen ein anderes Verständnis von der Aufgabe eines demokratischen Parlaments durchgesetzt hatte. In dem herrschenden Verständnis sollten die Fraktionen ihre Vorschläge einander übermitteln ,,mit dem Ziel, eine fraktionsübergreifende Gesetzesvorlage zu erarbeiten“. Etwaige Einwände würden ,intern diskutiert“, und dann könnte man „mit dem gemeinsamen Ergebnis in die Öffentlichkeit“ gehen ${ }^{49}$. So war 2005 bei der ersten Direkte-Demokratie-Reform - der Einführung von Bürgerentscheiden auf Bezirksebene - verfahren worden, und die Protagonisten sparten dazu nicht mit großen Worten: Es seien ,hier in Sachen politische Kultur und eines Projektes für das ganze Haus Maßstäbe gesetzt worden“" ${ }^{\text {‘50 }}$. Entsprechend sah der SPD-Unterhändler C. Gaebler bei der nachgerade „perfekt" - einmütig gelungenen Reform der direkten Demokratie auf Landesebene „wirklich eine große Stunde des Parlaments “51. Daß dieses Verständnis von parlamentarischer Demokratie vornehmlich bei den Regierungsparteien SPD und Linkspartei.PDS gepflogen wurde, die ihre Aufgabe ganz pragmatisch als Organisation von Mehrheiten verstanden, liegt auf der Hand; bemerkenswerterweise wurde es aber auch von der oppositionellen FDP geteilt. Umgekehrt vertrat die CDU - ihrer Funktion als große Oppositionsfraktion im Abgeordnetenhaus entsprechend jenes andere Verständnis, das statt auf das Management der Macht auf deren Kontrolle durch Öffentlichkeit setzte. Sie forderte, politische Vorschläge ,in den Ausschüssen, insbesondere im Rechtsausschuß, und in den Fraktionen“" zu diskutieren $^{52}$.

Im Plenum des Abgeordnetenhauses thematisiert wurden diese unterschiedlichen Verständnisse im November 2005, nachdem die Grünen einen Antrag „Mehr Demokratie für Berlinerinnen und Berliner auch auf Landesebene“ einge-

Abg. A. Hahn (FDP), Erklärung zur Abstimmung nach $§ 72$ GO, AH 18.5.2006, S. 7430 D - 7431 D. Hahn lehnte die beiden Hauptpunkte des Pakets - den Übergang zum Kanzlerprinzip und die Erleichterung der direkten Demokratie - ab. Was letztere anbelangt, war und blieb Hahn ein - skeptischer - Befürworter von Volksabstimmungen. Aber gegen die Tendenz der vorgesehenen Änderung hielt er weiterhin einen Themenausschluß für erforderlich und plädierte er dafür, die Quoren hoch zu legen (S. $7431 \mathrm{C}$ ). Vgl. Abg. Lederer (Linkspartei.PDS), AH 24.11.2005, S. 6550 C.

50 Vgl. a.a.O., S. 6551 B.

51 AH, 4.5.2006, S. 7343 C.

52 Vgl. Abg. M. Braun (CDU) - stellvertretender Fraktionsvorsitzender -, AH 24.11.2005, S. 6549 B. 
bracht hatten ${ }^{53}$. Als Oppositionspartei fühlten sie sich grundsätzlich dem Prinzip Öffentlichkeit verpflichtet und nahmen für sich in Anspruch, mit ihrem Vorstoß „einen politischen Prozeß zu befördern“54. Aus der Sicht der drei anderen Fraktionen hingegen, mit denen zusammen sie in der erwähnten Ad-hoc-Koalition die Demokratiereform auf Bezirksebene durchgesetzt hatten und mit denen sie jetzt wieder „Verständigungsgespräche“ führten ${ }^{55}$, begingen die Grünen Vertrauensbruch und handelten kontraproduktiv. Man warf ihnen „Vorpreschen“ vor $^{56}$ und daß sie sich ,auf Kosten eines Projekts, das man als überfraktionelle Runde miteinander hätte machen können“, profilieren würden ${ }^{57}$. Sie könnten ihrem Anliegen einen „Bärendienst“ erweisen ${ }^{58}$ und hätten womöglich „,den ersten Sargnagel in dieses Projekt geschlagen“59. „Eine ziemlich unseriöse und unredliche Sache“, gifteten die Machttechniker ${ }^{60}$ - ein ,gestörtes Verhältnis zum Parlamentarismus“, scholl es von der Opposition zurück ${ }^{61}$. Der Zusammenstoß schmerzte die Beteiligten noch im August 2006 - Monate nach der einmütigen Verabschiedung des zweiten Reformgesetzes ${ }^{62}$.

Nun kann es mit der Beschreibung dieser beiden Verständnisse nicht sein Bewenden haben. Eine Erklärung: Die erwähnte rasche Verabschiedung hing mit dem - freilich selbstverursachten - enormen Zeitdruck zusammen, unter dem der

53 „Mehr Demokratie für Berlinerinnen und Berliner auch auf Landesebene“ mit Entwurf eines „7. Gesetzes zur Änderung der Verfassung von Berlin“, Drs. 15/4472 v. 16.11.2005.

54 Abg. Ratzmann (Bündnis 90/Die Grünen), AH 24.11.2005, S. 6551 A.

55 Vgl. die Beschreibung des Abg. Ritzmann (FDP): „Wir haben hier eine Atmosphäre, eine Arbeitsebene, in der sich fraktionsübergreifend fast zwei Jahre lang Strukturen etabliert haben, die am Ende ein Ergebnis zeigten - eine Verfassungsänderung. Darauf können wir aufbauen.“ A.a.O., S. 6552 A. Ähnlich ders., AH 4.5.2006, S. 7347 A: „Wir haben zwei Jahre zusammengesessen. Davon haben wir jetzt im Nachhinein profitiert. Wir hatten eine funktionierende Arbeitsebene."

56 Vgl. Abg. Zimmermann (SPD), AH 24.11.2005, S. 6547 C.

57 Vgl. Abg. Lederer (Linkspartei.PDS), AH 24.11.2005, S. 6551 BC.

58 Vgl. a.a.O., S. 6550 D.

59 Vgl. Abg. Ritzmann (FDP), AH 24.11.2005, S. 6552 A, mit dem Argument, die Grünen verföchten die aus seiner Sicht, ,etwas irre Idee, die in dieser Frage mehr als wankende SPD jetzt pro Volksentscheide prügeln zu können, indem man im Alleingang einen Gesetzentwurf einbringt", ebenda.

60 Vgl. Abg. Lederer (Linkspartei.PDS), a.a.O., S. 6551 C.

61 Vgl. Abg. Braun (CDU), a.a.O., S. 6552 C.

62 Vgl. U. Schulte, SPD mustert die Grünen aus, in: taz Nr. 8048 v. 15.8.2006: „Übel haben alle den Grünen ihr Vorgehen beim Gesetz für stärkere Volksbegehren genommen. Während die Geschäftsführer - wegen großer Bedenken bei CDU und SPD - ein vorsichtiges Herantasten an einen Kompromiß hinter den Kulissen vereinbart hatten, brachten die Grünen im November 2005 einen fertig formulierten Antrag ins Parlament ein. ,Mit so einer Taktik torpediert man leicht eine Einigung. Schließlich müssen alle anderen plötzlich über ein öffentlich vorgehaltenes Stöckchen springen', sagt FDPler [FDP-Geschäftsführer Steffen] Saebisch.“ 
ganze Reformprozeß stand. Nur wenn das Parlament das Änderungsgesetz am 18. Mai in zweiter Lesung endgültig beschloß, waren die gesetzlichen Fristen für die nachfolgende Volksabstimmung einzuhalten, die zusammen mit den Wahlen zum Abgeordnetenhaus stattfinden sollte. Und eine Konsequenz: Das oben gebrauchte Adjektiv „,eigenartig“ zielt auf ein Transparenzproblem, das ja real bestand. Das Achte Gesetz zur Änderung der Verfassung von Berlin ist in der Tat ein Paradebeispiel dafür, wie das parlamentarische Verfahren, das doch gerade dazu geschaffen ist, den politischen Entscheidungsprozeß durch Öffentlichkeit $\mathrm{zu}$ rationalisieren und damit zu legitimieren, denaturiert wird, wenn die Volksvertreter wie eine verschworene Clique handeln. Hierfür genügten zwei Arrangements. Erstens: Indem die Akteure die Kompromißverhandlungen aus den parlamentarischen Gremien mit ihren Transparenzvorkehrungen, welche die Verfassung, die parlamentarische Geschäftsordnung und auch die Tradition getroffen hatten, auslagerten in eine völlig informell arbeitende interfraktionelle Arbeitsgruppe, gelang die perfekte Abschottung ${ }^{63}$. Zweitens: Die Einbindung aller Fraktionen beraubte die parlamentarische Debatte ihrer Substanz.

Nun soll hier keinen Illusionen vollkommener Transparenz parlamentarischer Entscheidungsprozesse nachgehangen werden. Selbstverständlich sind Vorformungen und Festlegungen in frühen Stadien und außerhalb der Volksvertretung genuiner Bestandteil der politischen Arbeit. Aber ein bestimmtes Maß an Durchschaubarkeit gewährleistet das parlamentarische Verfahren üblicherweise schon. Der Wechsel von Rede und Gegenrede zu einer Sache, von Kritik und Verteidigung einer Entscheidung, hat doch - und sei er noch so sehr der politischen Dramaturgie geschuldet - einen gewissen aufklärerischen Effekt. Dies gilt auch bei Verfassungsänderungen, für welche die Verfassung ja mit dem Erfordernis einer Zweidrittelmehrheit der gewählten Abgeordneten (Art. 100 S. 1 VvB) einen weitgehenden Konsens verlangt. Der rechnerisch kleine Schritt von der qualifizierten Mehrheit zur Einmütigkeit - so anerkennenswert die politische Leistung ist, eine derart hohe parlamentarische Zustimmung für die Reform der direkten Demokratie in Berlin zu organisieren - bedeutet jedoch eine andere Qualität des parlamentarischen Verfahrens wie der Beziehung der politischen Klasse zum Volk. Das Verfahren wandelt sich so, wie es für Entscheidungen des Parlaments in eigener Sache typisch ist ${ }^{64}-$ und in der Tat gehört die Chancenzutei-

63 So kritikwürdig dieses Vorgehen erscheint, so ,,bewährt“ ist es doch in der Parlamentsgeschichte. Man denke an die Rolle des „Fünfer-“ bzw. „Siebener-Ausschusses“ bei der Entstehung des Grundgesetzes 1948/49: Politische Effektivität setzte sich hier geradezu brutal gegen alle demokratischen Tugenden durch. Vgl. nur für eine spezielle Problematik Jung, Grundgesetz (Fn. 2), S. $278 \mathrm{ff}$.

64 Vgl. O. Jung, Direkte Demokratie als Gegengewicht gegen Kartelle der herrschenden Klasse? In: J. Wieland (Hrsg.), Entscheidungen des Parlaments in eigener Sache. Tagungsband zum Kolloquium anlässlich des 70. Geburtstages von Professor Dr. Hans 
lung für einen konkurrierenden Gesetzgeber zu dieser Kategorie von Entscheidungen. Wenn sich alle einig sind im Hohen Haus, wenn man sich gegenseitig auf die Schultern klopft und sich hütet, jemandem verbal wehzutun, ist für die interessierte Öffentlichkeit aus einer solchen Veranstaltung nicht mehr viel zu gewinnen $^{65}$. Im Verhältnis zum Volk ist der Preis jenes völligen Konsenses eine gewisse Entpolitisierung. Auf diese Problematik ist im Zusammenhang des nachfolgenden Referendums zurückzukommen.

\subsection{Eine Bürgerbewegung für mehr direkte Demokratie}

Eine Besonderheit des Reformprozesses bestand darin, daß neben der etablierten politischen Klasse eine weitere politische Kraft tätig war, die „von unten“ für eine Reform stritt. In ihrer Mitte stand die Bürgeraktion Mehr Demokratie e.V., die aber schon bald, nachdem die politische Situation zwischen den Parteien plastisch geworden war, ein „Bündnis für Direkte Demokratie“ zustande gebracht hatte $^{66}$. Von den elf teilnehmenden Organisationen sind neben Mehr Demokratie besonders zu erwähnen die Initiative Berliner Bankenskandal, die Humanistische Union, attac Berlin und der Bund der Steuerzahler Berlin; Gespräche mit der Gewerkschaft Erziehung und Wissenschaft ${ }^{67}$ hingegen hatten zu keinem Erfolg geführt. Am 10. März 2006 trat das Bündnis mit einer Pressekonferenz an die Öffentlichkeit. Die Sprecher - Dr. M. Efler von Mehr Demokratie e.V., H.J. Lindemann von der Initiative Berliner Bankenskandal und Dr. C. Bruch von

Herbert von Arnim am 19. März 2010, Berlin 2011 (Beiträge zum Parlamentsrecht Bd. 71), S. 81-102 (81 ff.).

65 Der Abg. Ratzmann (Bündnis 90/Die Grünen) spürte immerhin, daß es ,,schon etwas seltsam [ist], wenn wir anfangen, uns alle gegenseitig zu loben“, um dann gleich in einen müden Scherz auszuweichen, , aber dies ist vielleicht auch einmal eine Neuerung für dieses Haus.“ AH, 4.5.2006, S. 7345 A.

66 Als Katalysator für dieses Bündnis wirkte das Urteil, mit dem der Verfassungsgerichtshof am 22.11.2005 den Einspruch der Vertrauenspersonen des Volksbegehrens „Schluß mit dem Berliner Bankenskandal!“ gegen die Nichtzulassung ihres Projekts zurückwies (siehe Fn. 19). Lindemann, eine der Vertrauenspersonen, argumentierte nun: „Damit die Volksgesetzgebung nicht endgültig ad absurdum geführt wurde und wird, kann die Konsequenz nur lauten, den Haushaltsvorbehalt der Berliner Verfassung zu ändern." Unter Hinweis auf die bisher nicht umgesetzte einschlägige Vereinbarung im Koalitionsvertrag rief er dazu auf, Druck zu machen: „Kann es ein Bündnis von Vielen - mit Ihnen/Euch, weiteren Bürgerinitiativen und Organisationen usw. - für mehr Demokratie geben?“ Bankenskandal-Newsletter v. 11.12.2005. - Bereits am 20.12.2005 wurde dann das „Bündnis für Direkte Demokratie“ gegründet.

67 Vgl. Rundbrief Volksabstimmung - Newsletter der Bürgeraktion Mehr Demokratie e. V., Landesverband Berlin-Bandenburg - Nr. 1/06 v. 16.1.2006. 
der Humanistischen Union ${ }^{68}$ - stellten einen Gesetzentwurf des Bündnisses für „faire“ Volksbegehren und Volksentscheide ${ }^{69}$ vor. Drei Tage später überreichten sie Parlamentspräsident $W$. Momper diesen Entwurf.

Was die Volksgesetzgebung anbelangt, griff das Bündnis Reformstrukturen auf, die schon die Grünen in ihrem Gesetzentwurf vom November $2005^{70}$ vorgeschlagen hatten und die nachher im Zusammenhang der parlamentarischen Reform zu betrachten sind. Deutlich weiter als der Grünen-Entwurf ging das Positionspapier des Bündnisses insofern, als es auch ein obligatorisches Verfassungsreferendum - aus Bayern und Hessen bekannt und durchaus bewährt ${ }^{71}-$ sowie ein fakultatives Gesetzesreferendum vorschlug; letzteres freilich muß, da für dieses Instrument nur rudimentäre deutsche Erfahrungen vorliegen ${ }^{72}$, als Import aus der Eidgenossenschaft eingeschätzt werden. Überhaupt ist festzuhalten, daß das Bündnis zwar weitgehende, aber nicht unerhörte Vorstellungen vertrat - alles war aus den Regelungen anderer (Bundes-)Länder bzw. aus dem Kommunalverfassungsrecht bekannt. Man könnte sogar von einem gewissen Nachholcharakter sprechen: Da war keine Spur von Utopie im wörtlichen Sinne des (noch) nirgendwo Existierenden. Eine echte Innovation wurde der Berliner Politik nicht einmal von dieser Seite angesonnen. Methodisch mag gegen eine solche $\mathrm{Zu}$ sammenstellung von Forderungen mancher mit dem einschlägigen Urteil des Thüringer Verfassungsgerichtshofs von 2001 den Vorwurf erheben, daß sie ,jeweils die ,Rosinen herauspickt ${ }^{\text {““73 }}$. Die Kritisierten werden vermutlich in der

68 Die beteiligten Organisationen und die Sprecher des Bündnisses sind aufgeführt unter http://www.du-entscheidest-mit.de/809.99.html (Zugriff 12.8.2006).

69 Vgl. Bündnis für Direkte Demokratie, Entwurf eines Gesetzes zur Änderung der Verfassung von Berlin (9.3.2006), http://www.du-entscheidest-mit.de/fileadmin/berlin/pdf/gesetzentwurf-direkte-demokratie-berlin.pdf (Zugriff 8.9.2006). Dazu gab es eine zweiseitige Begründung („Der Gesetzentwurf des Bündnisses für Direkte Demokratie“).

70 Drs. $15 / 4472$ v. 16.11 .2005 .

71 Vgl. zur Praxis $O$. Jung, Regieren mit dem obligatorischen Verfassungsreferendum: Wirkung, Konterstrategie, Nutzungsversuche und Umgangsweise, in: ZParl 36 (2005), S. 161-187.

72 Zum Regelwerk und der geringen Praxis auf Reichsebene zur Weimarer Zeit vgl. C. Schwieger, Volksgesetzgebung in Deutschland. Der wissenschaftliche Umgang mit plebiszitärer Gesetzgebung auf Reichs- und Bundesebene in Weimarer Republik, Drittem Reich und Bundesrepublik Deutschland (1919-2002), Berlin 2005 (Tübinger Schriften zum Staats- und Verwaltungsrecht Bd. 71), S. 94-100; zur Ergänzung sowie zur ebenfalls sehr geringen Praxis in Rheinland-Pfalz vgl. O. Jung, Wenn der Souverän sich räuspert ... Vorwirkungen direktdemokratischer Korrekturmöglichkeiten, dargestellt an Beispielen aus Nordrhein-Westfalen, Niedersachsen und Rheinland-Pfalz, in: JzStVWiss 8 (1995), S. 107-176 (146-157, 172-175).

73 ThürVerfGH, Urt. v. 19.9.2001, LVerfGE 12, 405-463 (442). - Vgl. zu diesem Urteil bündig P.M. Huber, Entwicklung des Landesverfassungsrechts in Thüringen, in: JöR 52 (2004), S. 323-345 (340-342), sowie - methodologisch abschließend - F. Wittreck, Direkte Demokratie und Verfassungsgerichtsbarkeit. Eine kritische Übersicht zur deutschen 
Kombination bekannter und bewährter Regelungsmodule eine Optimierung der rechtlichen Rahmenbedingungen für direkte Demokratie sehen.

Eine Woche nach dem ersten Auftreten lud das Bündnis für den 27. März zu einer Podiumsdiskussion ins DGB-Haus ein. Der Bündnissprecher Bruch hielt einen Einführungsvortrag (,Mehr direkte Demokratie für Berlin“74), und dann diskutierten unter der Leitfrage „Wie viel Demokratie wollen wir wagen?“ je ein Vertreter der fünf Fraktionen des Abgeordnetenhauses - darunter vier der fünf Unterhändler der interfraktionellen Arbeitsgruppe ${ }^{75}$-, ferner der Experte Dr. J. Rux ${ }^{76}$ und Efler als Vertreter von Mehr Demokratie. Vor fast 100 Bürgerinnen und Bürgern zeigte sich eine interessante Differenzierung zwischen den teilweise radikaldemokratisch argumentierenden kleinen Oppositionsparteien Bündnis 90/Die Grünen und FDP sowie der Linkspartei.PDS einerseits und den eher skeptischen Vertretern der beiden Volksparteien andererseits.

Von den weiteren Aktivitäten des Bündnisses ist die wissenschaftliche Begleitung des Reformprozesses hervorzuheben, die C. Posselt ${ }^{77}$, Pressesprecher des Landesverbandes Berlin-Brandenburg von Mehr Demokratie, zusammen mit Efler leistete. Die auf der Website des Bündnisses vorgestellten Analysen des Diplom-Politologen:

- $\quad$ eine Kritik der bisherigen Situation, was direkte Demokratie angeht, in Ber$\operatorname{lin}^{78}$

- $\quad$ eine Übersicht über die bislang unternommenen neun Anläufe für Volksinitiativen bzw. Volksbegehren in Berlin mit Angaben zu Ziel, Trägern und Verlauf sowie Links $^{79}$,

- $\quad$ eine Synopse der geltenden Regelungen, des Reformkompromisses der Fraktionen des Abgeordnetenhauses und der Forderungen des Bündnisses ${ }^{80}$,

Verfassungsrechtsprechung in Fragen der unmittelbaren Demokratie von 2000 bis 2002, in: JöR 53 (2005), S. 111-185 (151-169).

74 Die erweiterte Fassung dieses Vortrags steht unter http://www.humanistische-union. de/fileadmin/hu_upload/berlin/2006/2006.03.27_Bruch_Vortrag.direkte.Demokratie.pdf (Zugriff 8.9.2006).

75 Lediglich für die CDU war nicht ihr Unterhändler Henkel, sondern der stellvertretende Fraktionsvorsitzende Gram erschienen.

76 Rux hatte in Tübingen die juristische Habilitationsschrift „,Direktdemokratische Verfahren im Parteienstaat. Rechtsgrundlagen und Rechtswirklichkeit der unmittelbaren Demokratie in Deutschland“" vorgelegt (gedruckt 2008 u.d.T.: Direkte Demokratie in Deutschland [Fn. 11]).

77 Jahrgang 1977, Mitglied des Bundesvorstands von Mehr Demokratie e.V. 2004-2006.

78 Unter http://www.du-entscheidest-mit.de/803.99.html (Zugriff 12.8.2006).

79 Unter http://www.du-entscheidest-mit.de/802.99.html (Zugriff 12.8.2006).

80 Unter http://www.du-entscheidest-mit.de/fileadmin/berlin/pdf/uebersicht.pdf (Zugriff 10. 9.2006). 
- ein hypothetisches Rechenspiel „Was wäre, wenn ... die bisherigen Volksentscheide in Deutschland nach dem geplanten Berliner Recht erfolgt wären? ${ }^{* 81}$ und

- ein ausführlicher Kommentar zur beschlossenen Reform („,Berlin wird demokratischer" $)^{82}$,

stellten eine Expertise bereit, die, soweit ersichtlich, noch für keine einschlägige Reform - und Reformen des Regelwerks für direkte Demokratie standen ja in den letzten Jahren in einigen Bundesländern an - so prompt und so gediegen geliefert wurde.

Schließlich ist Eflers ${ }^{83}$ Rolle zu unterstreichen. Der Diplomvolkswirt und -sozialökonom hatte sich die Sporen 1997/98 als Vertrauensmann der beiden Volksbegehren „Mehr Demokratie in Hamburg“ verdient ${ }^{84}$. Später machte Efler für Mehr Demokratie Lobbyarbeit in Brüssel, und nach seiner Übersiedlung nach Berlin übernahm er es, die Verbindungen zu den Parteien im Abgeordnetenhaus zu pflegen, was ihm, wie noch zu zeigen sein wird, vortrefflich gelang.

Dies ist das Stichwort für eine wichtige Beobachtung: Was das Bündnis für Direkte Demokratie, Mehr Demokratie und Efler unternahmen, war keine Massenmobilisierung, sondern klassische Lobbyarbeit, der Versuch einer Bürgerinitiative, die Entscheidungsträger des etablierten repräsentativ-demokratischen Systems politisch im eigenen Sinne zu beeinflussen. Mehr Demokratie versuchte diesmal also nicht, sich gleich dem Baron Münchhausen direktdemokratisch ,am eigenen Schopf aus dem Sumpf zu ziehen“, wie man es 1994 und 2000 in Bayern, 1997/8 in Hamburg, 1999 in Nordrhein-Westfalen, 2000 in Bremen, 2000/1 in Thüringen und auch in Berlin 1999 schon einmal versucht hatte ${ }^{85}$. Indes gilt es dabei zu bedenken, daß wegen des Berliner Verfassungstabus ein Vorgehen wie in den genannten anderen Bundesländern ausgeschlossen war. Es soll jetzt auch nicht ironisiert werden, die Basisdemokraten von Mehr Demokratie hätten nun den Stein der Weisen im klassischen Lobbyismus gefunden. Interessanterweise bewogen nämlich auch jene zunächst gescheiterten Vorstöße bereits relativ kurze

81 Unter http://www.du-entscheidest-mit.de/fileadmin/berlin/pdf/was_waere_wenn.pdf (Zugriff 10.9.2006).

82 Unter http://www.du-entscheidest-mit.de/fileadmin/berlin/pdf/kommentar_zur_reform. pdf (Zugriff 10.9.2006).

83 Jahrgang 1970; Mitglied des Bundesvorstands von Mehr Demokratie e. V. 1998-2004 und wieder seit Mai 2006.

84 Vgl. M. Efler, Der Kampf um Mehr Demokratie in Hamburg, in: H.K. Heußner/O. Jung (Hrsg.), Mehr direkte Demokratie wagen. Volksbegehren und Volksentscheid: Geschichte - Praxis - Vorschläge, 1. Aufl. München 1999, S. 205-222; auch in: H.P. Bull (Hrsg.), Fünf Jahre direkte Bürgerbeteiligung in Hamburg - unter Berücksichtigung von Berlin und Bremen, Hamburg 2001, S. 77-87.

85 Vgl. O. Jung, Grundsatzfragen der direkten Demokratie, in: Kost, Direkte Demokratie (Fn. 16), S. 312-366 (356 ff.). 
Zeit später die etablierten politischen Kräfte zu einschlägigen Konzessionen, wie die Reformen der direkten Demokratie in Hamburg $2001^{86}$, in NordrheinWestfalen $2002^{87}$ und in Thüringen $2003^{88}$ belegen; allein in Bayern ließ sich aus noch zu erforschenden Gründen kein solcher Effekt beobachten. Daß schließlich Lobbying den Erfolg brachte, besagt eben nicht, daß die vorangegangenen basisdemokratischen Aktionen (Unterschriftensammlungen für Volksbegehren und nachfolgende Verfassungsprozesse ${ }^{89}$ ) verfehlt gewesen wären. Näher liegt die Annahme, daß jener Druck ,von unten“ erst die Voraussetzung schuf, daß die Vertreter von Mehr Demokratie als Lobbyarbeiter überhaupt für voll genommen wurden.

Von Lobbying läßt sich hier freilich nur hinsichtlich der Art und Weise der Einflußnahme sprechen. Als Idealverein war Mehr Demokratie keine Wirtschaftslobby, die Wachstum und die Schaffung von Arbeitsplätzen verspricht, wenn die Politik ihr folgt, und mit dem Verlust beider droht, wenn jene nicht auf sie hört. Ebensowenig war Mehr Demokratie eine Betroffenen-Lobby, die - etwa im Falle von Großprojekten - klagt, wie hart ihre Mitglieder geschädigt würden, und zivilen Ungehorsam sowie den Gang durch alle juristischen Instanzen ankündigt. Mehr Demokratie, der kleine, „macht“lose Verein - 18 Jahre alt, gut 4.500 Mitglieder, etwa 600.000 Euro Haushalt (2005) - konnte nur seine Expertise, den Wissensvorsprung auf einem Politikfeld, das „,normalen“ Politikern in Deutschland immer noch recht fremd ist, einsetzen und dazu freilich auf die $\mathrm{Zu}$ stimmung der Bevölkerung verweisen: sei es indiziell durch ausnehmend positi-

86 Vgl. Efler, Kampf (Fn. 84), S. 86 f.; M. Schmidt, Die politische Debatte um eine Reform der Volksgesetzgebung 1997/1998 aus der Sicht der Hamburgischen Bürgerschaft, in: Bull, Bürgerbeteiligung (Fn. 84), S. 88-95 (95); R.-D. Klooß, Die Reform der Volksgesetzgebung 2001: Niedrigere Quoren und mehr Verfahrensflexibilität, a.a.O., S. 96-100.

87 Vgl. P. Neumann, Reform der sachunmittelbaren Demokratie in der Verfassung des Landes Nordrhein-Westfalen, in: NWVB1. 17 (2003), S. 1-8.

88 Edinger verwendet in seiner Analyse jenes Volksbegehrens denn auch das Oxymoron des „erfolgreichen Scheiterns“, vgl. M. Edinger, Die Herausforderung der repräsentativen Demokratie in Thüringen. Hintergründe, Verlauf und Wirkungen der Kontroverse um das Volksbegehren, in: K. Schmitt (Hrsg.), Herausforderungen der repräsentativen Demokratie, Baden-Baden 2003 (Veröffentlichungen der Deutschen Gesellschaft für Politikwissenschaft [DGfP] Bd. 20), S. 121-156 (129-138).

89 Siehe in diesem Zusammenhang den Vorwurf von durchaus wohlmeinender Seite, die Bürgeraktion Mehr Demokratie, welche die meisten einschlägigen Projekte gestartet hatte, habe durch „,radikale“ Gesetzentwürfe die negativen Urteile der Landesverfassungsgerichte im Grunde provoziert und so letztlich kontraproduktiv gehandelt (vgl. P. Neumann, Die Entwicklung der Rechtsprechung zu Volksbegehren und Volksentscheid nach der Deutschen Einheit, in: T. Schiller/V. Mittendorf [Hrsg.], Direkte Demokratie - Forschungsstand und Perspektiven, Wiesbaden 2002, S. 115-152 [147 f.], und dazu Jung, Grundsatzfragen [Fn. 85], S. 337 f.). 
ve Umfragewerte ${ }^{90}$, sei es erhärtet durch die Unterschriftenzahlen bei verschiedenen Volksbegehren ${ }^{91}$ bzw. die Zustimmung zu vorgeschlagenen Projekten bei Volksentscheiden ${ }^{92}$.

Aber brachte wirklich das Lobbying von Mehr Demokratie den Erfolg? Blieb der Kompromiß, auf den sich die Fraktionen verständigten, nicht allzu weit hinter den Wünschen und Vorstellungen dieser Bürgeraktion zurück? Wäre es nicht denkbar, daß die beschriebenen Vorstöße des Bündnisses nur sozusagen nebenherliefen? Wie ist hier überhaupt „Erfolg“ zu definieren, und wie läßt sich dieser nachweisen? Die vorletzte Frage ist relativ leicht zu beantworten. Erfolg kann hier vernünftigerweise nur Einflußnahme und Drängen in die gewünschte Richtung bedeuten. Daß die Bürgeraktion ihre Ziele nicht voll erreicht hat, mindert den Wert solcher Beeinflussung nicht; alles unterhalb 100prozentiger Durchsetzung als Mißerfolg zu qualifizieren wäre fundamentalistisch. Die letzte Frage dagegen - fand eine solche maßvolle Einflußnahme tatsächlich statt? - bedarf

90 Schon lange wünscht sich nach allen Umfragen eine überwältigende Mehrheit der Deutschen Volksentscheide (innerdeutsche Differenzen in diesem Zusammenhang zeigt und erklärt M. Klein, „Was bleibt von der friedlichen Revolution? Plebiszitäre Orientierungen im vereinten Deutschland“ in: H. Meulemann [Hrsg.], Werte und nationale Identität im vereinten Deutschland. Erklärungsansätze der Umfrageforschung, Opladen 1998, S. 155 176). - $\mathrm{Zu}$ diesen und späteren Umfragen vgl. O. Jung, Direkte Demokratie. Die Angst der politischen Klasse vor dem Volk: Fortschritte und Rückschritte in den letzten 15 Jahren in Deutschland, in: H.H. v. Arnim (Hrsg.), Defizite in Staat und Verwaltung. Beiträge auf der 10. Speyerer Demokratietagung vom 25. und 26. Oktober 2007 an der Deutschen Hochschule für Verwaltungswissenschaften Speyer, Berlin 2010 (Schriftenreihe der Hochschule Speyer Bd. 203), S. 105-171 (155 f.). - Siehe jüngst die repräsentative Studie des Meinungsforschungsinstituts TNS-Emnid im Auftrag der Bertelsmann-Stiftung (Juni 2011), http://www.bertelsmann-stiftung.de/bst/de/media/xcms_bst_dms_34119_34120_2. pdf und http://www.bertelsmann-stiftung.de/bst/de/media/xcms_bst_dms 34121 34144 2.pdf (Zugriff jeweils 28.7.2011), und die einläßliche Untersuchung von L.P. Feld/ $\bar{Z}$. Hessami/L. Reil: Direkte Demokratie in der Bundesrepublik Deutschland? - Ergebnisse einer Umfrage zur Einführung direkter Volksrechte auf Bundesebene, in: JdD 2 (2011), S. 107-134.

91 Gerade Efler konnte darauf verweisen, daß „seine“ Volksbegehren in Hamburg 1998 die zweit- bzw. vierthöchste bislang in deutschen Bundesländern erzielte Zustimmung erreicht hatten: Nach Nordrhein-Westfalen 1978 („Kooperative Schule“) mit 29,8 Prozent ein Ausnahmefall - (vgl. O. Jung, Daten zu Volksentscheiden in Deutschland auf Landesebene [1946-1992], in: ZParl 24 [1993], S. 5-13 [9]) folgen Hamburg 1998 (,Volksgesetzgebung“) mit 18,4 Prozent, Thüringen 2000 (,Volksgesetzgebung“) mit 18,3 Prozent und wieder Hamburg 1998 (,Bezirklicher Bürgerentscheid“) mit 18,1 Prozent (vgl. Jung, Grundsatzfragen [Fn. 85], S. 356 f.).

92 Siehe vor allem die Fälle echter Volksgesetze, in denen volksbegehrte Entwürfe an den Urnen über parlamentarische Konkurrenzvorlagen obsiegten, so erstmals in Bayern 1995 (,Kommunaler Bürgerentscheid“, vgl. O. Jung, Der Volksentscheid über die Einführung des kommunalen Bürgerentscheids in Bayern am 1. Oktober 1995, in: JzStVWiss 9 [1996], S. 191-272 [232]), ferner 1998 wiederum in Bayern („Abschaffung des Senats“), in Schleswig-Holstein (,Rechtschreibreform“) und Hamburg (,Bezirklicher Bürgerentscheid“) sowie erneut in der Hansestadt 2004 (,Wahlrechtsreform“). 
zunächst der methodologischen Vorklärung. Einen „echten“ Nachweis mögen vielleicht später einmal Historiker führen, wenn ihnen die Akten zur Verfügung stehen. Bis dahin können nur bescheidenere Anforderungen gestellt werden. Insbesondere die Pauschalurteile von Beteiligten halten der Quellenkritik nicht stand: So würdigte der bereits erwähnte Pressesprecher Posselt den zwischen den Fraktionen erzielten Kompromiß als einen „Riesenerfolg“ für das Bündnis bzw. Mehr Demokratie ${ }^{93}$; aber das kann die Wissenschaft natürlich nicht zum Nennwert nehmen. Und wenn Parlamentspräsident Momper (SPD) dem Bündnis mündlich bescheinigte - wie die Gelobten alsbald kolportierten -, es habe „einen entscheidenden Anteil am Zustandekommen der Reform gehabt ${ }^{\text {‘9 } 94}$, so ist das eine freundlich-konventionelle Rede, aber gewiß keine „belastbare“ Aussage. Auch die von der Bürgeraktion berichtete spannende Dramaturgie - demnach war noch im März zu erwarten, „daß es höchstens ein bescheidenes Reförmchen der Parteien geben wird, wenn überhaupt!““95; dann sei ,unter unserem Druck ... in den Fraktionen eine Diskussion in Gang“" gekommen ${ }^{96}$ und gerade in den letzten Wochen habe Mehr Demokratie ,noch wichtige Verbesserungen durchsetzen“" können ${ }^{97}$ - erscheint plausibel, aber ist (noch) nicht wissenschaftlich belegbar. Es sind in der Tat mehr Einzelbeobachtungen und Verfahrensdetails, die einen Einfluß im behaupteten Sinne indizieren:

- Wenn die SPD bei der I. Lesung unverhohlen davon sprach, daß in der Öffentlichkeit ,im Vorfeld ein großer Druck aufgebaut“" worden sei ${ }^{98}$, hatte die Arbeit von Mehr Demokratie bzw. des Bündnisses in der Öffentlichkeit offenbar gewirkt.

- Auf fällt, wie selbstverständlich die Redner im Parlament Einschätzungen und Forderungen von Mehr Demokratie als Referenzgrößen benutzten, ob es da um die Stellung Berlins in einem Direkte-Demokratie-Ranking der Bun-

93 Vgl. Mehr Demokratie e.V. [Pressesprecher], Einladung zum Treffen der Berliner und Brandenburger Aktivisten v. 13.4.2006; Rundbrief Volksabstimmung Nr. 3/06 v. 28.4.2006.

94 Rundbrief Volksabstimmung Nr. 3[richtig: 4?]/06 v. 2.6.2006.

95 Bankenskandal-Newsletter v. 17.3.2006.

96 Vgl. http://www.du-entscheidest-mit.de/789.html (Zugriff 12.8.2006); auch in: mehr demokratie 17 (2006), H. 2 (Nr. 70), S. 24.

97 Rundbrief Volksabstimmung Nr. 3/06 v. 28.4.2006.

98 Abg. Gaebler (SPD), AH 4.5.2006, S. 7344 A. 
desländer ging ${ }^{99}$ oder um die Stellungnahme von Mehr Demokratie zum Kompromiß der Fraktionen ${ }^{100}$.

- Ferner ist das Klima der Zusammenarbeit hervorzuheben. Daß zum Hearing des Bündnisses am 27. März Vertreter aller fünf Fraktionen erschienen, war nach Berliner Usancen nicht selbstverständlich.

- Ein starkes Indiz für den ,guten Draht“, den der Hauptlobbyist Efler zu den Fraktions-Unterhändlern hatte, stammt schon aus der Debatte des Vorjahres über die erste Demokratiereform dieser Legislaturperiode, die Einführung von Bürgerentscheiden auf Bezirksebene. Hier wies während der II. Lesung der PDS-Redner auf den Verein Mehr Demokratie hin und fuhr fort: ,... den ich herzlich begrüße und bei dem ich mich schon jetzt für seine aktive Begleitung des Prozesses herzlich bedanke“101. Und am Ende dieser Lesung schloß der FDP-Redner seinen Beitrag wie folgt: „Ganz zum Schluß möchte ich mich bei ,Mehr Demokratie" bedanken, besonders bei Dr. Michael Efler. Für die gute Zuarbeit, für die gute Beratung: Herzlichen Dank!، ${ }^{102}$ Eine solche positive Aufnahme der Expertise des Fachverbands für direkte Demokratie war bei keiner anderen einschlägigen Reform bislang zu beobachten. Eine bessere Voraussetzung, um jetzt auch bei der zweiten Demokratiereform, der Erleichterung der Volksgesetzgebung auf Landesebene, auf die Abgeordneten einzuwirken, ließ sich kaum denken, und die Chance wurde allem Anschein nach genutzt ${ }^{103}$.

Nachdem die Reform das Abgeordnetenhaus passiert hatte, versuchte das Bündnis in einem Kommentar zu klären, wie es dazu kam, und nannte drei Ursachen:

Vgl. Abg. Lederer (Linkspartei.PDS), a. a. O., S. 7341 D. - Lederer bezog sich offenbar auf Mehr Demokratie e.V. (Hrsg.), 1. Volksentscheid-Ranking. Die direktdemokratischen Verfahren der Länder und Gemeinden im Vergleich, Berlin 2003. Siehe dazu R. Kampwirth, Volksentscheide in Deutschland: Bayern Spitze, Berlin Schlusslicht, in: S. Brink/ H.A. Wolff (Hrsg.), Gemeinwohl und Verantwortung. Festschrift für Hans Herbert von Arnim zum 65. Geburtstag, Berlin 2004, S. 367-373.

100 So erklärte der SPD-Unterhändler Abg. Gaebler in der I. Lesung, er sei „sehr froh“ darüber, daß Mehr Demokratie in ihrem neuesten Anschreiben für das politisch geschnürte Paket werbe, und „sehr dankbar“ dafür, daß dort gesagt werde, daß dies nur ein weiterer Schritt sei, AH 4.5.2006, S. 7344 C.

101 Abg. Peter-Rudolf Zotl (PDS), AH 16.6.2005, S. 5948 C. - Dazu vermerkt das Protokoll: „Beifall bei der PDS, der SPD, den Grünen und der FDP“, a.a.O.

102 Abg. Ritzmann (FDP), a.a.O., S. 5951 A. - Nach einem weiteren, letzten Satz (,Nun sollten wir abstimmen und Berlin etwas demokratischer machen, als es bisher war.") vermerkt das Protokoll: „Beifall bei der FDP, der SPD, der PDS und den Grünen“, a.a.O.; es ist anzunehmen, daß dieser auch dem Dank an Mehr Demokratie und Efler galt.

103 Zwar richtete der Abg. Gaebler (SPD) in der I. Lesung seinen Dank vergleichsweise allgemein auch an ,jene, die außerhalb des Parlaments die Angelegenheit begleitet haben. Hier sind wichtige Anregungen gekommen, auch wenn nicht alles berücksichtigt werden konnte“, AH 4.5.2006, S. 7344 D - 7345 A. Doch damit dürfte er ebenfalls Mehr Demokratie und Efler gemeint haben. 
- die positiven Erfahrungen, welche die Berliner Politik - einschließlich der bislang immer skeptischen CDU - mit der gestärkten Direktdemokratie auf Bezirksebene (Einführung des Bürgerentscheids) seit einem Jahr gemacht habe ${ }^{104}$;

- die Paketlösung als Instrument des Ausgleichs und der Vereinigung von Interessen;

- den öffentlichen Druck, den das Bündnis mit Aktionen und Veranstaltungen erzeugt habe; dies habe die beteiligten Parteien „zur Einigung in einigen zentralen Streitpunkten der Reform“ bewogen ${ }^{105}$.

Als Beobachter wird man diesen Erklärungen voranstellen, daß sich in der Berliner Politik offenkundig ein kalpós aufgetan hatte: Festgefügte Strukturen in den Parteien waren aufgebrochen, neue Alterskohorten hatten im Parlament das Sagen übernommen, und ein anderer Ansatz, Politik zu treiben, hatte Einzug gehalten - auf letzteres ist noch zurückzukommen.

Die erste Erklärung ist anachronistisch. Als Mitte Januar 2006, wie oben gezeigt, das entscheidende Paket für die Reform der direkten Demokratie auf Landesebene geschnürt wurde, waren erst vier Bürgerbegehren gestartet, aber noch keines abgeschlossen ${ }^{106}$. Zum ersten Bürgerentscheid in Berlin [-Lichtenberg] kam es am 17. September 2006, zeitgleich mit dem Referendum über die Verfassungsänderung ${ }^{107}$. Die zweite Erklärung könnte man zuspitzen, daß der oben be-

104 Vgl. zur Illustration die Ausführungen des Abg. Henkel (CDU), AH 4.5.2006, S. 7343 A: „Gerade machen wir in Berliner Bezirken unsere Erfahrungen mit den geschaffenen Instrumenten direkter Bürgerbeteiligung. Ich denke dabei insbesondere an meine Kreuzberger Freunde, die mit gutem Zuspruch ein Bürgerbegehren gegen die Umbenennung der Kochstraße in Rudi-Dutschke-Straße zu laufen haben."

105 Berlin wird demokratischer. Ein Kommentar zur Reform von Volksinitiative, Volksbegehren und Volksentscheid, http://www.du-entscheidest-mit.de/fileadmin/berlin/pdf/kommentar_zur_reform.pdf (Zugriff 10.9.2006).

106 Vgl. Efler/Posselt, Direkte Demokratie (Fn. 17), S. 296-302, mit Tabelle S. 300.

107 F. Decker, Der Königsweg führt in die Irre, in: Berliner Republik 8 (2006), H. 5, S. 79-82 (79), entgeht dieser Anachronismus, wenn er vermutet, die Bereitschaft der Parteien, besonders von CDU und SPD, zum Umdenken, ,dürfte hauptsächlich an den positiven Erfahrungen liegen, die man mit dem plebiszitären Instrument auf der kommunalen Ebene gemacht hat". Umso mehr Rückenwind habe die Diskussion bekommen, ,,als die neu geschaffenen Rechte (sc. Bürgerbegehren und Bürgerentscheid) lebhaft genutzt wurden". Zunächst wurde das Bürgerbegehren in den Berliner Bezirken nicht 2005, wie Decker offenbar meint - ein geläufiger Irrtum (siehe oben Fn. 17) -, sondern schon 1978 eingeführt, aber eben imperfekt gestaltet. Gewiß lag nahe, daß das Instrument mit der Einführung des Bürgerentscheids 2005 neuen Schwung bekommen würde, aber es lagen dazu eben noch keine Erfahrungen vor. Obendrein setzt Decker die Akzente falsch: Die (virtuellen) positiven Erfahrungen mit kommunaler Direktdemokratie erscheinen ihm vorrangig (,hauptsächlich“), der eigentliche Beweggrund der Stärkung der Position des Regierenden Bürgermeisters durch Übergang zum Kanzlerprinzip hingegen nachrangig (,hinzu kommt"). - Auch der Abg. Henkel (CDU) hatte, wie sich erweisen sollte, vorschnell geurteilt. Das Anliegen des von ihm erwähnten Bürgerbegehrens gegen die (teilweise) Um- 
schriebene „Deal“ im repräsentativ-demokratischen System eben vorzüglich „paßte“. Zur dritten Erklärung muß es vorerst bei der vorsichtigeren Feststellung verbleiben, wonach es starke Indizien dafür gibt, daß Mehr Demokratie bzw. das Bündnis dank ihrer engen Kooperation mit der interfraktionellen Arbeitsgemeinschaft durch Druck und Geschick die Reform der direkten Demokratie in Berlin mit-vorantrieben.

\subsection{Die Volksabstimmung über die Reform}

Über jene Teile des Achten Gesetzes zur Änderung der Verfassung von Berlin, welche die direkte Demokratie betrafen, mußte nach Art. 100 S. 2 VvB noch eine Volksabstimmung stattfinden. Dieses mit der Verfassung von 1995 eingeführte spezielle Verfassungsreferendum stellte politisch-psychologisch die Aufarbeitung des Amputationstraumas von 1974 dar: Nie wieder sollte das Volk als Gesetzgeber durch einen Beschluß seiner Vertreter einfach entmachtet werden können. Da die Klausel aber neutral formuliert war und schlechthin für jedwede Änderung der Art. 62 (Volksbegehren) und $63 \mathrm{VvB}$ (Volksentscheid) galt ${ }^{108}$, griff sie auch im vorliegenden Falle, in dem sowohl nach der subjektiven Intention der Akteure als auch nach dem objektiven Gehalt der verabschiedeten Regelung die Verfassungsänderung auf eine Erleichterung der Volksgesetzgebung gerichtet war. Damit kam es in Berlin zu der wohl singulären Situation, daß das Volk auf Befragen seiner Vertretung darüber entscheiden sollte, ob es selbst mehr Volksrechte haben wolle. Um den Unterschied noch einmal zu verdeutlichen: Daß ein Parlament an dieser Stelle dem Drängen der Basis nachgibt und den Druck ,von unten“ sozusagen in Verfassungsnormen gießt, die das Volk dann noch ratifiziert, ist der oft zu beobachtende Normalfall ${ }^{109}$. Aber in Berlin war die Initiative eindeutig „von oben“ gekommen, und der Druck, den man im Verlaufe des Reformprozesses feststellen kann, war der Druck einer Lobby, aber nicht der „Massen“ der Stadt. Dann über das Ergebnis noch einen Volksentscheid abzuhalten - dabei konnten einen schon eigenartige Gefühle beschleichen ${ }^{110}$.

benennung der Kochstraße in Rudi-Dutschke-Straße sollte beim Bürgerentscheid am 21. Januar 2007 ,echt“ scheitern, d. h. eine Mehrheit an den Urnen votierte gegen die begehrte Rückbenennung und damit für den Status quo der Umbenennung, vgl. Mehr Demokratie e. V., Zweiter Berliner Demokratiebericht, 2008, S. 28, $49 \mathrm{f}$.

108 Vgl. H. Lemmer in: Pfennig/Neumann (Fn. 5), Art. 100 Rn. 3.

109 Vgl. F. Decker, Regieren im „Parteienbundesstaat“. Zur Architektur der deutschen Politik, Wiesbaden 2011, S. 168: „Fast immer handelte es sich um Konzessionen der herrschenden Eliten an die Bevölkerung, die unter massivem Druck zustande kamen.“

110 Diese schienen auch in einigen seltsamen Schlagzeilen der Presse durch: „Einmaliges Vertrauen in das Volk“, in: taz Nr. 7974 v. 18.5.2006 - das erinnerte doch an das berühmte Brecht-Gedicht nach dem 17. Juni 1953; „Das Volk darf sich bald mehr Macht 
Massives Unbehagen dagegen erzeugten die Umstände dieser Volksabstimmung. Das begann bereits mit der beschriebenen intransparenten und im Grunde entpolitisierenden Art und Weise, wie das Abgeordnetenhaus jene Reform verabschiedete. Damit wurde nicht nur der Sinn des parlamentarischen Verfahrens verfehlt; die Volksvertretung versäumte es auch, die politischen Voraussetzungen für die folgende Volksabstimmung zu schaffen. Am Beispiel der Volksgesetzgebung ist schon herausgearbeitet worden, daß es sich um ein sekundäres Verfahren handelt und deshalb nicht ,unversehens Probleme auf ein ahnungsloses Publikum ein(stürzen)““. Auszugehen ist vielmehr von einer längeren, kontroversen Behandlung des Problems im parlamentarischen Primärverfahren. Angenommen wird ,eine politische Vorklärung ..., die Entwicklung unterschiedlicher Lösungsmodelle, das Ausloten von Kompromißmöglichkeiten sowie ein Herausarbeiten des jeweiligen Pro und Contra, von denen das Publikum in dem nunmehrigen Volksgesetzgebungsverfahren profitiert“111. Was bei der Volksgesetzgebung nur als politisch-psychologischer Zusammenhang erscheint, ist beim Referendum institutionell verklammert. Aber wie sollen denn die Bürgerinnen und Bürger bei der Volksabstimmung zwischen Ja und Nein entscheiden, wenn ihre Vertreter die Mühe „der verantwortungsvollen Grundsatzkleinarbeitung،(112 _ hier: der Stärkung der direkten Demokratie - gewissermaßen im Geheimen geleistet haben? Nur wenn die Volksvertreter ihrerseits das Pro und Contra einer Reform eingehend öffentlich debattiert haben, kann das Volk sich sozusagen „wohlberaten“ entscheiden, ob es sich dem mehrheitlichen Votum seiner Vertreter anschließt oder dieses, wie die Minderheit im Parlament empfohlen hat, verwirft. Anders bei dem in Berlin praktizierten Vorgehen. Mangels Transparenz und bei fehlender Diskursivität trat das Parlament, vertreten durch seinen Präsidenten $^{113}$, dem Volk funktional als Obrigkeit gegenüber, die von den Bürgerin-

geben“, in: Berliner Zeitung Nr. 116 v. 19.5.2006 - ging nicht laut Grundgesetz alle Staatsgewalt von diesem Volke aus?

111 Vgl. O. Jung, Volksgesetzgebung in Deutschland, in: Leviathan 15 (1987), S. 242-265 (250).

112 Vgl. A. Greifeld, Volksentscheid durch Parlamente. Wahlen und Abstimmungen vor dem Grundgesetz der Demokratie, Berlin 1983 (Studien und Gutachten aus dem Institut für Staatslehre, Staats- und Verwaltungsrecht der Freien Universität Berlin H. 12), S. 42.

113 Unmittelbar nach der Abstimmung am Ende der (debattenlosen) II. Lesung hielt Parlamentspräsident Momper (SPD) eine kurze Ansprache voll freundlicher Allgemeinheiten. Durch die eben beschlossene Verfassungsänderung würden „die Möglichkeiten direkter Demokratie für die Berlinerinnen und Berliner ausgeweitet. Wir wollen die Quoren für Volksabstimmungen und Volksentscheide senken, auch um der Politikverdrossenheit entgegenzuwirken. Den Bürgerinnen und Bürgern Berlins wird es dadurch wesentlich erleichtert, politische und gesellschaftliche Entscheidungsprozesse unmittelbar mitzugestalten oder Gesetze auf den Weg zu bringen, so daß die repräsentative Demokratie um das plebiszitäre Element sinnvoll ergänzt wird.“ AH 18.5.2006, S. 7430 BC. 
nen und Bürgern eine plebiszitäre Akklamation zu der von ihr getroffenen, aber überhaupt nicht vermittelten Entscheidung verlangte.

Verstärkt wurde das Unbehagen durch die öffentliche Wahrnehmung der Verfassungsänderung insgesamt, die sich nahezu vollständig auf den Wechsel vom Senats- zum Kanzlerprinzip konzentrierte: „Durchbruch im Parlament: Mehr Macht für Wowereit“114 und „Regierender wird mächtig wie ein Ministerpräsi-

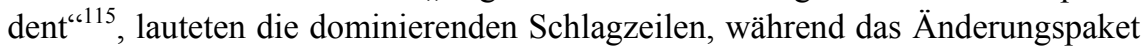
geschnürt wurde, und darin drückte sich offensichtlich die Perspektive der politischen Elite aus. Die Reform der direkten Demokratie trat weitgehend in den Hintergrund.

Nun sind Parlamentsdebatten gewiß nicht die einzige politische Informationsquelle. So wie die Parteien einen Wahlkampf führen, könnte natürlich auch ein Abstimmungskampf vor dem Volksentscheid die politische Aufklärung über das zu lösende Sachproblem fördern. Aber hier zeigte sich wieder das Elend des „Von oben“ ausgelösten Referendums: Angesichts der Einmütigkeit des Parlaments fühlte sich im Grunde keine Partei bemüßigt, etwas Größeres zu tun, um die Bürgerinnen und Bürger für die Reform zu gewinnen. Nun ist ja von anderen Bundesländern her bekannt, daß die politischen Repräsentanten vor obligatorischen Referenden kein Interesse an einer Abstimmungsdebatte haben ${ }^{116}$; völlig unerwartet war das Verhalten der politischen Elite Berlins also nicht. Aber es verblüffte denn doch, wie tief die kommende Volksabstimmung und deren Gegenstand von der etablierten Politik gehängt wurden. Die größere Regierungspartei rang sich in ihrer Parteizeitung zwei Sätze zu der „Wahl mit Besonderheiten“ ab: „Die Berliner SPD hat sich dafür eingesetzt, Volksinitiativen, Volksbegehren und Volksentscheide zu erleichtern. Die erfolgreiche Volksabstimmung am 17. September ist aus Sicht der SPD ein weiterer Baustein, um die Teilhaberechte

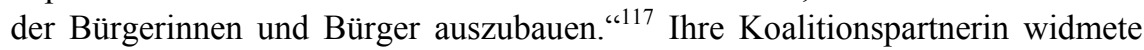
der bevorstehenden Volksabstimmung immerhin ein Plakat mit dem schlichten Text: „Stimmt. Beim Volksentscheid am 17. September mit Ja für mehr direkte Demokratie. Damit Unsinn verhindert werden kann. “118 Auch die Grünen ${ }^{119}$ setz-

114 Berliner Morgenpost Nr. 88 v. 29.3.2006; ähnlich Berliner Morgenpost v. 19.5.2006; Die Welt Nr. 116 v. 19.5.2006.

115 Tsp. Nr. 19175 v. 28.4.2006.

116 Vgl. Jung, Verfassungsreferendum (Fn. 71), S. 181-184.

117 Vorwärts Berlin, 9/2006, S. II. - Im Internet präsentierte die SPD allerdings noch ,Informationen zur Volksabstimmung": eine ausführliche Darstellung des Inhalts der Reform, http://www.berlinwahl.spd.de/servlet/PB/menu/1684023/index.html (Zugriff 13.9.2006).

118 Das Plakat ist wiedergegeben unter: http://www.linkspartei-berlin.de/wahlen/wahl_2006/ volksabstimmung/ (Zugriff 13.9.2006). 
ten einen Text ins Internet. Indes erscheint der Einzelnachweis solcher Spurenelemente eines Abstimmungskampfes müßig. Der beherrschende Eindruck war, daß das Thema Volksabstimmung im Parteienwettbewerb des August/September 2006 in Berlin, in der öffentlichen - sinnlichen - Werbung auf den Straßen und Plätzen der Stadt, praktisch nicht vorkam. Von der politischen Psychologie her wirkte es schon befremdlich, daß die politische Elite Berlins ausgerechnet zu mehr direkter Demokratie sozusagen auf leisen Sohlen gelangen wollte.

Bei trug zu diesem Verhalten der Parteien gewiß das Regelwerk für die Volksabstimmung. Die politischen Profis in Senat und Abgeordnetenhaus hatten für diesen akklamationsähnlich gedachten Akt „natürlich“ keine Quorenfalle aufgestellt, wie sie Initiativen ,von unten“ zugemutet wurde ${ }^{120}$ und in der sie sich selbst womöglich hätten fangen können. Das für die Volksabstimmung verabschiedete spezielle Gesetz bestimmte vielmehr eindeutig, daß die Zustimmung zur Änderung der Artikel 62 und 63 der Verfassung von Berlin erteilt sei, ,,wenn mehr als die Hälfte der gültigen Stimmen die Abstimmungsfrage bejaht ${ }^{\text {“121 }}$. So erzdemokratisch diese Regel ist, verursachte das angelegte „Zweierlei Maß“ doch ein ungutes Gefühl ${ }^{122}$.

Dabei war diese Reform kein Selbstläufer. Am 13. August widmete der „Tagesspiegel“" seine beliebte Pro-und-Contra-Argumentation, bei der man dann telephonisch votieren darf, der (etwas „schräg“ formulierten) Frage: „Soll die direkte Mitbestimmung erleichtert werden? ${ }^{\text {“123 }}$ Das natürlich nicht repräsentative Ergebnis: 58,4 Prozent der Anrufer sprachen sich für die Reform und 41,6 Prozent dagegen aus ${ }^{124}$. Hier drohte vor allem der mitregierenden Linkspartei.PDS ein politisches Desaster. Es war nämlich nicht so - wie es manchmal mißver-

119 http://72.14.221.104/search?q=cache:swHIO4NMMn0J:gruene-berlin.de/site/fileadmin /datei-en/2006/wahlzeitung_2006.pdf + grProzentC3ProzentBCne+berlin+landesverband + volksabstimmung\&hl $=\mathrm{de} \& \mathrm{gl}=\mathrm{de} \& \mathrm{ct}=\mathrm{clnk} \& \mathrm{~cd}=13$ (Zugriff 14.9.2006).

120 Bisher ein 50prozentiges Beteiligungs- oder aber ein 33prozentiges Zustimmungsquorum (Art. 63 Abs. 2 VvB a.F.), künftig ein 25prozentiges Zustimmungsquorum (Art. 63 Abs. 1 S. 3 VvB n.F.), dabei natürlich immer die Mehrheit der Abstimmenden.

$121 \S 6$ S. 1 Gesetz über die Durchführung der Volksabstimmung nach Art. 100 S. 2 der Verfassung von Berlin am 17. September 2006 v. 25.5.2006, GVB1. S. 448. - Diese Verfassungsbestimmung selbst macht insoweit keine Vorgaben.

122 Dieses zweierlei Maß läßt sich immer wieder zeigen, vgl. O. Jung, Das Quorenproblem beim Volksentscheid. Legitimität und Effizienz beim Abschluß des Verfahrens der Volksgesetzgebung, in: ZPol 9 (1999), S. 863-898 (879 f.). Entscheidend ist die Erwünschtheit bzw. Unerwünschtheit des jeweiligen Vorhabens aus gouvernementaler Perspektive.

123 Tsp. Nr. 19279 v. 13.8.2006. Auch die Contra-Argumentation war unglücklich, weil sie weniger die konkret zur Entscheidung stehende Reform als vielmehr das Prinzip der direkten Demokratie als solches - fundamentalistisch - angriff. - Nicht minder seltsam war der Begleitartikel überschrieben: L. v. Törne, Wenn das Volk sich selbst vertritt, a.a.O. als ob das Prinzip der Vertretung das A und $\mathrm{O}$ der Demokratie wäre.

124 Tsp. Nr. 19281 v. 15.8.2006. 
standen wurde ${ }^{125}$-, daß das gesamte Verfassungsänderungs-Paket dem Referendum unterworfen gewesen wäre. Das Änderungsgesetz formulierte rechtstechnisch klar: „Artikel I Nr. 8 tritt nur in Kraft, wenn in einer Volksabstimmung gemäß Artikel 100 Satz 2 der Verfassung von Berlin eine Mehrheit der Änderung der Artikel 62 und 63 der Verfassung von Berlin zustimmt. “" ${ }^{126}$ Damit zeichnete sich das Szenario ab, daß die nicht referendumspflichtigen Teile des Pakets - vor allem der die SPD am meisten interessierende Übergang zum Kanzlerprinzip - in Kraft träten, während die politische Kompensation für die Linkspartei.PDS (und die Grünen) bei der Volksabstimmung scheiterten ${ }^{127}$. Hier bestand für diese beiden Parteien das Risiko des „Reinfalls“; sie wären dann objektiv politisch ,geprellt“" gewesen.

In dieser Situation regten sich die zivilgesellschaftlichen Kräfte. Zunächst der Rundfunk Berlin-Brandenburg (rbb): Das „Inforadio“ sendete am 25. August ein Interview mit Parlamentspräsident Momper (SPD) („Volksentscheide in der Hauptstadt?") ${ }^{128}$, die „Abendschau“ brachte am 1. September einen informativen Beitrag über die Reform und „Radioeins“ am 15. September ein Interview mit einem Experten für direkte Demokratie in seinem Magazin „Die schöne Woche“. Der „Tagesspiegel“ ließ in der letzten Woche vor der Wahl einen Politikwissenschaftler mit einer pointierten Stellungnahme („,Der Irrweg der direkten Demokratie“) zu Wort kommen ${ }^{129}$. Die ,tageszeitung“ bemühte sich ebenfalls um die Aufbereitung der Reformproblematik ${ }^{130}$. Sachliche Informationen brachten in

125 Sogar im „Tagesspiegel“ hieß es nach der II. Lesung irrig: „Wenn die Volksabstimmung erfolgreich ist, darf der Regierungschef ab der nächsten Wahlperiode die Senatsmitglieder ernennen und entlassen und die Richtlinien zur Regierungspolitik eigenverantwortlich festlegen." $z a[=U$. Zawatka-Gerlach], Mehr Macht und mehr Demokratie, in: Tsp. Nr. 19195 v. 19.5.2006.

126 Art. II Abs. 2 S. 1 Achtes Gesetz zur Änderung der Verfassung von Berlin v. 25.5.2006, GVB1. S. 446.

127 In diesem Sinne mahnte der Abg. Ratzmann (Bündnis 90/Die Grünen) bei der I. Lesung zu einer ,gemeinsamen Anstrengung“ der Werbung für die Reform, damit „wir nicht nachher zwar die Richtlinienkompetenz haben, aber nicht mehr Demokratie“, AH 4.5.2006, S. 7345 D.

$128 \mathrm{http} / /$ www.inforadio.de/static/dyn2sta_article/835/138835_article.shtml (Zugriff 17.9. 2006). Siehe auch „Hintergrund zum Inforadio Spezial Volksabstimmung über Bürgerbeteiligung“ http://www.inforadio.de/static/dyn2sta_article/202/135202 article print.shtml (Zugriff 17.9.2006).

129 F. Decker, Der Irrweg der direkten Demokratie, in: Tsp. Nr. 19309 v. 12.9.2006. Die bereits angenommene Erwiderung eines Kollegen (des Verfassers dieses Beitrags) wurde - kleines Foul - im letzten Moment mit flauer Begründung abgesetzt und dafür, zwei Tage vor der Volksabstimmung, ,eine aktuelle Position zur Gesundheitsreform“ (!) gedruckt. Das Bündnis für Direkte Demokratie stellte daraufhin beide Texte - Deckers Stellungnahme und die Replik von O. Jung, Die praktische Vernunft spricht für „Ja“ - ins Netz unter http://www.du-entscheidest-mit.de/1265.html (Zugriff 16.9.2006).

130 Vgl. U. Schulte, Bürger haben Extra-Wahl, in: taz Nr. 8075 v. 15.9.2006; M. Wolf, Eine Wahl wie aus dem Lehrbuch, in: taz Nr. 8076 v. 16.9.2006. 
den letzten Tagen auch die „Welt“ („Mehr direkte Demokratie“"131, „Volksabstimmung über Volksabstimmung “(132) und wiederum der ,Tagesspiegel“ (,Mehr Demokratie wagen“"133).

Besonders energisch ging das „Bündnis für Direkte Demokratie“ gegen die in Berlin vorherrschende Nonchalance vor dieser Volksabstimmung an. Schon am 25. April, als die beiden Lesungen im Abgeordnetenhaus noch gar nicht „über die Bühne gegangen“ waren, aber der Verlauf des „Stücks“ schon absehbar war, betonte Efler den Willen des Bündnisses, daß die Berlinerinnen und Berliner am 17. September „eine bewußte Entscheidung treffen“ sollten, und skizzierte eine eigene Kampagne ${ }^{134}$. Vor der II. Lesung kritisierte das Bündnis den Verzicht auf eine Debatte als „nicht nachvollziehbar“; damit werde „,eine Chance vertan, die Öffentlichkeit zu informieren“"135. Als dann die offiziellen Informationen zur Volksabstimmung verschickt wurden, bezeichnete das Bündnis sie als ,unzureichend und teilweise irreführend“"136. Im Rahmen seiner eigenen Kampagne gab das Bündnis eine Broschüre heraus, die an 180.000 Haushalte verteilt wurde ${ }^{137}$; sie stand auch im Internet ${ }^{138}$. Im „Berliner Fenster“ (Fahrgastfernsehen in der UBahn) wurde geworben ${ }^{139}$. Ferner war es dem Bündnis gelungen, mehrere Prominente zu gewinnen, die für ein Ja zur Volksabstimmung warben: den Komödianten D. Hallervorden, den Schriftsteller W. Kaminer, die Publizistin L. Rosh, den ehemaligen Greenpeace-Chef T. Bode, den Vorsitzenden des DGB-Bezirks Berlin/Brandenburg, D. Scholz, die Bundesbeauftragte für die Unterlagen des Staatssicherheitsdienstes der ehemaligen DDR, M. Birthler, den Politikwissenschaftler P. Grottian (Freie Universität) und den Soziologen D. Rucht (Wissenschaftszentrum Berlin $)^{140}$. Worauf die Bündnis-Aktivisten „besonders stolz“ wa-

131 Die Welt Nr. 217 v. 16. 9. 2006.

132 Die Welt am Sonntag v. 17. 9. 2006.

133 Tsp. Nr. 19313 v. 16. 9. 2006.

134 „Unser Endspiel heißt: Volksabstimmung ...“ Spenden-Werbebrief von Mehr Demokratie v. 25.4.2006.

135 „Bündnis für Direkte Demokratie“, PM („Lautlos im Abgeordnetenhaus - Keine Debatte zu Demokratie-Reform im Parlament") v. 17.5.2006.

136 „Bündnis für Direkte Demokratie“, Presseinformation v. 7.8.2006; ätzend die Bemerkung, die Darstellung des Verfassungstextes - gegenübergestellt waren die geltende und die neue Fassung - habe ,den Charakter von Kleingedrucktem“.

137 Vgl. Infozeitung für mehr Demokratie, in: taz Nr. 8052 v. 19.8.2006. - Später wurde für die achtseitige Zeitung eine Auflage von 200.000 genannt, so Lindemann im Bankenskandal-Newsletter v. 17.9.2006.

138 bündnis für direkte demokratie: Du entscheidest mit! Informationen zur Volksabstimmung am 17.9.2006 http://www.du-entscheidest-mit.de/fileadmin/berlin/pdf/infozeitung. pdf (Zugriff 18.9.2006).

139 Vgl. Rundbrief Volksabstimmung Nr. 6/06 v. 29.8.2006; Lindemann im BankenskandalNewsletter v. 17.9.2006.

140 http://www.du-entscheidest-mit.de/1191.99.html (Zugriff 8.9.2006). 
ren $^{141}$, ließ sie freilich unter „friendly fire“ geraten: Die „tageszeitung“ lästerte über diesen „Wahlkrampf ${ }^{\text {s142 }}$. Daneben wurde auch konventionelle Arbeit mit Veranstaltungen und Interviews ${ }^{143}$ geleistet.

Inhaltlich hatte Efler am Tag der II. Lesung im Abgeordnetenhaus die Position des Bündnisses wie folgt umrissen: „Die Reform beseitigt nicht alle Probleme, unter denen die Volksgesetzgebung in der Vergangenheit gelitten hat. Und daß Volksbegehren zur Landesverfassung zwar formal zugelassen, aber mit so hohen Hürden versehen werden, daß sie praktisch ausgeschlossen bleiben, ist eigentlich eine Frechheit.“ Alles in allem sei die Reform jedoch ein Fortschritt gegenüber den bisherigen Regelungen. „Da es die Option ,Ja, aber ‘ bei einer Volksabstimmung nicht gibt, werden wir die Bürger um ihre Zustimmung bitten. “144

Das Urteil über diese Kampagne des Bündnisses fällt ambivalent aus. Einerseits ist es ein großartiges Zeichen von Engagement, daß ein so kleiner Trägerkreis aus eigenen Mitteln und mit eigener Kraft - selbstredend ohne irgendeine Erstattungsaussicht - eine solche Kampagne entwarf und durchführte, „nur“ um aus der von den Abgeordnetenhaus-Parteien beabsichtigten Akklamation eine „bewußte Entscheidung“ der Bürgerinnen und Bürger zu machen. Andererseits: Was für die kleine Schar der Aktivisten eine riesige Anstrengung bedeutete, war, bezogen auf die Millionenstadt und verglichen mit dem gleichzeitigen, Unsummen verschlingenden, professionell geführten Wahlkampf, eben doch fast nichts.

Volksabstimmung über die Neuregelung von Volksbegehren und Volksentscheid in der Verfassung von Berlin am 17. September 2006

\begin{tabular}{|c|c|c|c|c|c|c|c|c|c|c|c|c|c|}
\hline \multirow{4}{*}{$\begin{array}{c}\text { Räumliche } \\
\text { Einheit }\end{array}$} & \multirow{4}{*}{$\begin{array}{l}\text { Stimm } \\
\text { berech- } \\
\text { tigte }\end{array}$} & \multicolumn{2}{|c|}{ Wähler } & \multicolumn{2}{|c|}{$\begin{array}{l}\text { Abgegebene } \\
\text { Stimmen }\end{array}$} & \multicolumn{2}{|c|}{$\begin{array}{l}\text { Ungültige } \\
\text { Stimmenen }\end{array}$} & \multicolumn{6}{|c|}{ Gültige Stimmen } \\
\hline & & \multirow{3}{*}{ absolut } & \multirow{3}{*}{$\%$} & \multirow{3}{*}{ absolut } & \multirow{3}{*}{$\%$} & \multirow{3}{*}{ absolut } & \multirow{3}{*}{$\%$} & \multirow{2}{*}{\multicolumn{2}{|c|}{ Insgesamt }} & \multicolumn{4}{|c|}{ mit Votum } \\
\hline & & & & & & & & & & \multicolumn{2}{|l|}{$\mathrm{ja}$} & \multicolumn{2}{|l|}{ nein } \\
\hline & & & & & & & & absolut & $\%$ & absolut & $\%$ & absolut & $\%$ \\
\hline & 1 & 2 & 3 & 4 & 5 & 6 & 7 & 8 & 9 & 10 & 11 & 12 & 13 \\
\hline 1 Mitte & 190.550 & 103.924 & 54,5 & 102.302 & 98,4 & 5.683 & 5,6 & 96.619 & 94,4 & 80.471 & 83,3 & 16.148 & 16,7 \\
\hline $\begin{array}{l}2 \text { Friedrichs- } \\
\text { hain- } \\
\text { Kreuzberg }\end{array}$ & 165.331 & 92.451 & 55,9 & 91.284 & 98,7 & 5.311 & 5,8 & 85.973 & 94,2 & 73.456 & 85,4 & 12.517 & 14,6 \\
\hline 3 Pankow & 274.380 & 153.400 & 55,9 & 151.512 & 98,8 & 7.441 & 4,9 & 144.071 & 95,1 & 123.661 & 85,8 & 20.410 & 14,2 \\
\hline
\end{tabular}

141 Vgl. Rundbrief Volksabstimmung Nr. 6/06 v. 29.8.2006.

142 Vgl. taz, Nr. 8064 v. 2.9.2006.

143 Vgl. „Volksbegehren sind dann eher durchsetzbar“ [Interview mit M. Efler], in: Junge Welt Nr. 210 v. 11.9.2006.

144 Mehr Demokratie e.V., Bundesverband, Presseinformation Nr. 16/06 v. 18.5.2006. 


\begin{tabular}{|c|c|c|c|c|c|c|c|c|c|c|c|c|c|}
\hline $\begin{array}{l}4 \text { Charlotten- } \\
\text { burg- } \\
\text { Wilmersdorf }\end{array}$ & 216.374 & 139.582 & 64,5 & 136.833 & 98,0 & 7.197 & 5,3 & 129.636 & 94,7 & 106.433 & 82,1 & 23.203 & 17,9 \\
\hline 5 Spandau & 160.411 & 93.940 & 58,6 & 92.976 & 99,0 & 5.415 & 5,8 & 87.561 & 94,2 & 73.456 & 83,9 & 14.105 & 16,1 \\
\hline $\begin{array}{l}6 \text { Steglitz- } \\
\text { Zehlendorf }\end{array}$ & 213.787 & 146.857 & 68,7 & 144.986 & 98,7 & 6.761 & 4,7 & 138.225 & 95,3 & 111.754 & 80,8 & 26.471 & 19,2 \\
\hline $\begin{array}{l}7 \text { Tempelhof- } \\
\text { Schöneberg }\end{array}$ & 231.249 & 145.139 & 62,8 & 142.920 & 98,5 & 7.473 & 5,2 & 135.447 & 94,8 & 112.811 & 83,3 & 22.636 & 16,7 \\
\hline 8 Neukölln & 193.014 & 107.180 & 55,5 & 105.927 & 98,8 & 6.065 & 5,7 & 99.862 & 94,3 & 84.145 & 84,3 & 15.717 & 15,7 \\
\hline $\begin{array}{l}9 \text { Treptow- } \\
\text { Köpenick }\end{array}$ & 193.936 & 111.443 & 57,5 & 110.499 & 99,2 & 5.149 & 4,7 & 105.350 & 95,3 & 91.158 & 86,5 & 14.192 & 13,5 \\
\hline $\begin{array}{l}10 \text { Marzahn- } \\
\text { Hellersdorf }\end{array}$ & 201.209 & 99.139 & 49,3 & 99.157 & 99,0 & 4.032 & 4,1 & 94.125 & 95,9 & 81.099 & 86,2 & 13.026 & 13,8 \\
\hline $\begin{array}{l}11 \text { Lichten- } \\
\text { berg }\end{array}$ & 201.096 & 99.905 & 49,7 & 98.728 & 98,8 & 7.380 & 7,5 & 91.348 & 92,5 & 77.185 & 84,5 & 14.163 & 15,5 \\
\hline $\begin{array}{l}12 \text { Reinik- } \\
\text { kendorf }\end{array}$ & 184.143 & 114.794 & 62,3 & 112.785 & 98,2 & 5.992 & 5,3 & 106.793 & 94,7 & 88.741 & 83,1 & 18.052 & 16,9 \\
\hline Berlin & 2.425 .480 & 1.407 .754 & 58,0 & 1.388 .909 & 98,7 & 73.899 & 5,3 & 1.315 .010 & 94,7 & 1.104 .370 & 84,0 & 210.640 & 16,0 \\
\hline Berlin-Ost & 1.012 .351 & 544.933 & 53,8 & 538.959 & 98,9 & 28.068 & 5,2 & 510.891 & 94,8 & 437.408 & 85,6 & 73.483 & 14,4 \\
\hline Berlin-West & 1.413 .129 & 862.821 & 61,1 & 849.950 & 98,5 & 45.831 & 5,4 & 804.119 & 94,6 & 666.962 & 82,9 & 137.157 & 17,1 \\
\hline
\end{tabular}

Nach: Der Landeswahlleiter (Hrsg.): Wahlen in Berlin. Abgeordnetenhaus. Bezirksverordnetenversammlungen. Volksabstimmung über die Neuregelung von Volksbegehren und Volksentscheid in der Verfassung von Berlin in Berlin am 17. September 2006. Endgültiges Ergebnis. Berlin 2006, S. 98. Die Prozentzahlen in den Spalten 3, 4 und 5 beziehen sich auf Spalte 1; die in Spalte 9 auf Spalte 4, die in den Spalten 11 und 13 auf die 8.

An der Volksabstimmung beteiligten sich 1.407 .754 von 2.425.480 Stimmberechtigten (58,0 Prozent). 73.899 Stimmen (5,3 Prozent) waren ungültig. Von den gültigen 1.315.010 Stimmen lauteten 1.104.370 (84,0 Prozent) auf „Ja“ und 210.640 (16,0 Prozent) auf Nein. Da mehr als die Hälfte der gültigen Stimmen die Abstimmungsfrage bejaht hatten, war nach dem Durchführungsgesetz die Zustimmung zur Änderung der Art. 62 und 63 VvB erteilt. Der Regierende Bürgermeister gab das Ergebnis der Volksabstimmung im Gesetz- und Verordnungsblatt für Berlin bekannt und stellte zugleich fest, daß die Verfassungsänderung in Kraft trete ${ }^{145}$. Am 26. Oktober 2006 wurde die Reform der direkten Demokratie in Berlin wirksam ${ }^{146}$.

Hätten Senat und Abgeordnetenhaus die Regeln für diese Volksabstimmung nicht rein erfolgsorientiert aufgestellt, d.h. auf ein Quorum verzichtet, wäre -

145 Bek. über das Ergebnis der Volksabstimmung am 17. September 2006 über die Neuregelung von Volksbegehren und Volksentscheid in der Verfassung von Berlin v. 12.10.2006, GVB1. S. 1002.

146 Die Änderung trat laut Art. II Abs. 1 und 2 S. 2 des Achten Gesetzes zur Änderung der Verfassung von Berlin i.V.m. der eben erwähnten Bek. mit Beginn der 16. Wahlperiode des Abgeordnetenhauses von Berlin in Kraft. Diese Wahlperiode begann mit dem ersten Zusammentritt des am 17. September 2006 neu gewählten Abgeordnetenhauses (vgl. Art. 54 Abs. 1 S. 2 VvB); dies war der 26. Oktober 2006. 
hypothetisch - festzuhalten: Das künftige Quorum für die verfassungsändernde Volksgesetzgebung: Zweidrittel-Mehrheit der Abstimmenden und zugleich Zustimmung von mindestens der Hälfte der Stimmberechtigten (Art. 63 Abs. 2 S. 3 VvB), wäre nach diesen Zahlen nur im ersten Teil erfüllt gewesen. Angesichts der niedrigen Abstimmungsbeteiligung von 58,0 Prozent entsprachen die 1.104.370 Ja-Stimmen aber nur 45,5 Prozent der 2.425.480 Stimmberechtigten ${ }^{147}$. Die Legitimation, welche diese Verfassungsänderung bei der Volksabstimmung am 17. September 2006 erfuhr, lag also deutlich niedriger als die Legitimation, die für künftige Verfassungsänderungen im Wege der Volksgesetzgebung gefordert wurde ${ }^{148}$.

Für die weitere Interpretation des Abstimmungsergebnisses ist zunächst in Erinnerung zu rufen, daß es in Deutschland - im Unterschied zur Schweiz - keine Abstimmungsforschung gibt auf dem Niveau der (freilich schon länger bestehenden) Wahlforschung ${ }^{149}$. Nähere Aufschlüsse bietet das Abstimmungsergebnis jedenfalls, wenn man es mit den gleichzeitig abgehaltenen Wahlen zum Abge-

147 Von daher trugen Erfolgsmeldungen der Art: „Mit 84 Prozent haben die Berlinerinnen und Berliner am 17. September Ja zur Verbesserung von Volksabstimmungen gesagt" (K. Flothmann, Absolute Mehrheit für direkte Demokratie! Ein Erfolg für Berlin: Volksabstimmung stimmte mit 84 Prozent Ja, in: mehr demokratie 17 [2006], H. 4 [Nr. 72], S. 6), nicht zur politischen Aufklärung bei. Nicht nachvollziehbar ist auch die gerade zitierte Schlagzeile „Absolute Mehrheit für direkte Demokratie!“ Bezogen auf die Zahl der Abstimmenden bzw. der gültigen Stimmen, war eine relative Mehrheit sowohl nach der Logik - eine einzige, nur mit Ja oder Nein zu beantwortende Frage - als auch nach der Verfahrensordnung ausgeschlossen; sollte die Reform Erfolg haben, brauchte sie - insoweit immer eine absolute Mehrheit. Bezog sich die Behauptung aber auf die Gesamtheit der Stimmberechtigten - nur so wird der triumphierende Duktus verständlich, und das paßt zu der Rede von 84 Prozent der Berlinerinnen und Berliner -, hatten die Verantwortlichen es versäumt nachzurechnen.

148 Zur grundsätzlich gebotenen Korrespondenz zwischen Verabschiedungs- und Änderungsquorum vgl. O. Jung, Die Landesverfassungsreferenden des Jahres 1994. Daten und Probleme, in: LKV 5 (1995), S. 319-321 (320 f.).

149 Vgl. O. Jung, Direkte Demokratie - Forschungsstand und Perspektiven, in: Schiller/Mittendorf, Direkte Demokratie (Fn. 89), S. 22-63 (39). - Symptomatisch dafür ist der Bericht des Landeswahlleiters mit dem Titel „Wahlen (sic!) in Berlin am 17. September 2006“: Auf 162 Seiten voller Ergebnisse, Tabellen, Kommentierungen, Grafiken und Karten bietet er für die Volksabstimmung an jenem Tage eine Tabelle (S. 98) und zum Ergebnis einen Satz: „Hierbei (sc. bei der Volksabstimmung) stimmte die große Mehrheit für eine Ausweitung der direkten politischen Mitbestimmung der Bürger" (S. 104). Jeder Kommentar, jede Auswertung fehlt. - Auch in der Wissenschaft verhielten sich manche konform, etwa im traditionellen Wahl-Aufsatz in der „Zeitschrift für Parlamentsfragen“: O. Niedermayer/R. Stöss, Die Berliner Abgeordnetenhauswahl vom 17. September 2006: Ein „Weiter so“ trotz herber Verluste des Koalitionspartners, in: ZParl 38 (2007), S. 84100 , erwähnten nur in einem einzigen Sätzlein in einer Fußnote das Faktum, daß „,neben“ der Wahl zum Abgeordnetenhaus ,eine Volksabstimmung zur Änderung der Verfassung statt(fand)“, S. 86 Fn. 16. 
ordnetenhaus $^{150}$ (Zweitstimmen) vergleicht und regional nach Berlin-Ost, BerlinWest sowie den Bezirken aufgliedert.

Bei den Wahlen zum Abgeordnetenhaus wurden 1.407.754 Zweitstimmen abgegeben und bei der Volksabstimmung ebenfalls genau 1.407.754 Stimmen. Das heißt, daß alle politisch Interessierten, die für die wichtigste Wahl den Weg ins Wahllokal auf sich genommen hatten, sich auch an der Volksabstimmung beteiligten; kein einziger Bürger, keine einzige Bürgerin war vor der Volksabstimmung sozusagen „von der Fahne gegangen“. Dieses Ergebnis ist nicht selbstverständlich. Bei den elf Referenden der Jahre 1991 bis 2003 in Bayern, Bremen, Hessen, Mecklenburg-Vorpommern und Thüringen, die gleichzeitig mit Europa-, Bundestags-, Landtags- oder Kommunalwahlen abgehalten wurden, lag die Abstimmungs- regelmäßig unter der Wahlbeteiligung ${ }^{151}$. Als 1995 über die revidierte Verfassung von Berlin abgestimmt wurde, lag die Abstimmungsbeteiligung um 1,2 Prozentpunkte hinter der Wahlbeteiligung zurück ${ }^{152}$. Angesichts dieser Ausgangslage erscheint es wenig sinnvoll, mit Blick auf die Volksabstimmung 2006 zu kritisieren, ,,auch diese prinzipielle Möglichkeit einer strukturellen Alternative zum Status quo politischer Entscheidungsstrukturen“ habe nicht zu einer höheren Wahlbeteiligung geführt ${ }^{153}$.

Die Zahl der ungültigen Stimmen stieg von 2,0 Prozent bei den Wahlen zum Abgeordnetenhaus (Zweitstimmen) auf 5,3 Prozent bei der Volksabstimmung und erklomm im Bezirk Lichtenberg das Maximum von 7,5 Prozent. Aber diese

150 Die Wahlen zu den Bezirksverordnetenversammlungen sind wegen des erweiterten Elektorats - auch 16- und 17jährige sowie EU-Bürgerinnen und -Bürger durften wählen nicht vergleichbar.

151 Vgl. für die Referenden 1994/95 Jung, Quorenproblem (Fn. 122), S. 888. - Bei den Volksabstimmungen 2002 in Hessen betrug der Rückstand auf die Beteiligung an der Bundestagswahl 0,5 Prozentpunkte, aber dieser Vergleich ist methodisch nicht korrekt wegen der unterschiedlichen Größe des Kreises der Wahl- bzw. Stimmberechtigten. 2003 lag in Bayern die Beteiligung an den Volksabstimmungen um 0,2 Prozentpunkte unter derjenigen bei der Landtagswahl. - Dies gilt für „von oben“ angesetzte Referenden. Bei Volksentscheiden, die ,,von unten“ im Wege der Volksgesetzgebung durchgesetzt wurden, ist der Abstand zwischen Wahl- und Abstimmungsbeteiligung oft noch viel größer.

152 Vgl. Statistisches Landesamt Berlin (Hrsg.), Wahlen im Oktober 95. Abgeordnetenhaus, Bezirksverordnetenversammlungen. Volksabstimmung über die Verfassung von Berlin. Endgültiges Ergebnis, o. O., o. J. (Berlin 1995).

153 Vgl. T. Rudek, Über das Siegerlächeln der Repräsentanten. Anmerkungen zur Krise der parlamentarischen Demokratie am Beispiel der Wahl zum Berliner Abgeordnetenhaus, http://www.linkezeitung.de/cms/content/view/1075/205/ (Zugriff 21.9.2006). Ob diese Wirkung überhaupt zu erwarten ist, erörtert $O$. Jung, ,Wahlbeteiligung runter, Bürgerbeteiligung rauf!“ - Zu welcher Art von Partizipation führt mehr direkte Demokratie? In: S. Frech/I. Juchler (Hrsg.): Bürger auf Abwegen? Politikdistanz und politische Bildung. Siegfried Schiele für seine Verdienste um die Didaktik politischer Bildung, Schwalbach/Ts. 2011, S. 51-80. - Immerhin läßt sich, nicht zuletzt mit Blick auf die Bezirke Marzahn-Hellersdorf und Lichtenberg, fragen, wie tief wohl die Wahlbeteiligung gefallen wäre, wenn es nicht gleichzeitig eine Volksabstimmung gegeben hätte. 
hohe Zahl ist durchaus keine Besonderheit. Der Vergleich zeigt, daß solche Werte bei Abstimmungen üblich sind. Bei den letzten vier Verfassungsreferenden in Bayern 1998 und 2003 lagen die Ungültigkeitsquoten zwischen 2,8 und 6,3 Prozent $^{154}$. Als 2002 über drei Verfassungsänderungen in Hessen abgestimmt wurde, stimmten 5,9, 6,4 bzw. 8,8 Prozent der Abstimmenden ungültig ${ }^{155}$. Offenbar muß man davon ausgehen, daß manche Fragestellungen etliche Bürgerinnen und Bürger überfordern, die dann für sich die „Lösung“ wählen, ungültig zu stimmen machttechnisch sozusagen „Enthaltung“. Dazu mag im jetzigen Berliner Fall die nicht gerade „,bürgerfreundliche“ Formulierung der Abstimmungsfrage beigetragen haben: „Stimmen Sie der Änderung der Artikel 62 und 63 der Verfassung von Berlin in der im Gesetz- und Verordnungsblatt für Berlin am 3. Juni 2006 auf den Seiten 446 und 447 veröffentlichten Fassung zu? ${ }^{\text {‘156 }}$ Hätte man nicht entsprechend der an die Stimmberechtigten versandten „Information zur Volksabstimmung“, wonach das „Ziel der neuen Regelung ... die Stärkung der direkten Demokratie" sei, weniger rechtstechnisch, aber dafür verständlicher fragen können: Stimmen Sie der Stärkung der direkten Demokratie, wie sie das Abgeordnetenhaus am 18. Mai 2006 beschlossen hat, zu? Gleichwohl sollte man nicht nur auf jene 5,3 Prozent der Abstimmenden starren, die hier wohl überfordert wurden, sondern zuerst begrüßen, daß 94,7 Prozent sich in dieser Sache durchaus eine Meinung gebildet hatten.

Schlüsselt man die Ja- und Nein-Stimmen nach Berlin-Ost und Berlin-West auf, zeigt sich eine klare Differenz: 85,6 Prozent gegen 14,4 Prozent im Ostteil liegt erheblich höher als 82,9 Prozent gegen 17,1 Prozent im Westteil der Stadt. Noch deutlicher wird die Scheidung auf der Bezirksebene. In SteglitzZehlendorf, übrigens dem Bezirk mit der höchsten Abstimmungsbeteiligung von 68,7 Prozent, stimmten nur noch 80,8 Prozent für und 19,2 Prozent gegen diese Reform. Umgekehrt bejahten sie in Treptow-Köpenick bei einer durchschnittlichen Abstimmungsbeteiligung von 57,5 Prozent, sogar 86,5 Prozent, während nur 13,5 Prozent sie ablehnten.

Da unter den gegebenen Umständen vor allem die Nein-Stimmen interessieren, kann man mit einem Kompliment beginnen. Wenn alle Parteien des Abge-

154 6,3 Prozent ungültige Stimmen beim Volksentscheid 2003 über das Verfassungsreformgesetz - Zusammentritt des Landtags nach der Wahl, Parlamentsinformation und Verankerung eines strikten Konnexitätsprinzips, http://www.volksentscheide2003.bayern. de/ve2003/990/gesamt1.html (Zugriff 21. 9. 2006).

155 8,8 Prozent ungültige Stimmen beim Volksentscheid 2002 über die Aufnahme des Konnexitätsprinzips, vgl. Hessisches Statistisches Landesamt (Hrsg.), Die Volksabstimmungen in Hessen am 22. September 2002. Endgültige Ergebnisse, Wiesbaden 2002 (Statistische Berichte B VII $4-02 / 2)$, S. 2.

156 Siehe http://www.statistik-berlin.de/wahlen/aghbvvwahl-2006/stimmzettel/sz-va.pdf (Zugriff 19.9.2006); ferner $\S 3$ Durchführungsgesetz. 
ordnetenhauses sowie eine rege Gruppierung der Zivilgesellschaft zu einer Abstimmung mit „Ja“ aufrufen und weit und breit niemand ist, der ein „Nein“ artikuliert und propagiert, zeugt es jedenfalls von einem eigenständigen politischen Kopf, wenn 210.640 Bürgerinnen und Bürger sich ihre eigenen Gedanken über die Sache machen und dabei zu einem anderen Urteil als die Vorgenannten kommen. Diese Zahlen widerlegen E. Fraenkels apodiktische Behauptung, unter einem parlamentarischen Regierungssystem bedeute ein Plebiszit bestenfalls „das Abhalten eines außerparlamentarischen Partei-Exerzierens، ${ }^{\text {“157 }}$. Daß die Vertretungskörperschaft so gut wie einstimmig eine Vorlage verabschiedet, führt keineswegs dazu, daß auch die Vertretenen einmütig zustimmen. Noch ein Referendum über die parlamentarisch beschlossene Neuregelung abzuhalten, war also durchaus nicht müßig ${ }^{158}$. Dieses Ergebnis enttäuschte auch die bereits mehrfach angesprochenen Akklamations-Erwartungen der Parteien des Abgeordnetenhauses, ungeachtet deren die Bürgerinnen und Bürger letztlich eine normale Volksentscheidung trafen.

Für die Frage nach der Motivation dieser Ablehnung mag die regionale Verteilung einen Hinweis geben: 19,2 Prozent stimmten in Steglitz-Zehlendorf gegen diese Reform, 17,9 in Charlottenburg-Wilmersdorf, 16,9 in Reinickendorf, 16,7 in Mitte und in Tempelhof-Schöneberg. Liegt da nicht der Gedanke einer Korrelation mit dem Verbreitungsgebiet des in dieser Frage bekannt skeptischen „Tagesspiegels“ nahe?

Selbst in den genannten Bezirken lag die Zustimmung zu dieser Reform aber noch weit höher als bei der bereits erwähnten „Tagesspiegel“-Leserbefragung fünf Wochen vor dem Abstimmungstag. Ob diese Differenz real und damit erklärungsbedürftig ist, steht dahin. Man könnte ja auch argumentieren, daß diese private Umfrage in einer Leserschaft, bei der naturgemäß nur die Aktiven votieren, nicht vergleichbar sei mit einer staatlich organisierten Volksabstimmung, bei der doch die staatsbürgerliche Erwartung dahin geht, daß die Bürgerinnen und Bürger von ihrem demokratischen Abstimmungsrecht Gebrauch machen. Unter-

157 E. Fraenkel, Die repräsentative und die plebiszitäre Komponente im demokratischen Verfassungsstaat, Tübingen 1958 (Recht und Staat in Geschichte und Gegenwart, H. 219/220), S. 29. - Ohnehin generalisierte Fraenkel hier, wie der Kontext zeigt - Illusorischmachen des Stimmgeheimnisses durch organisierten Abstimmungsboykott -, die negativen „Weimarer Erfahrungen“ mit dem Verfahren der Volksgesetzgebung, aber wertete z. B. nicht die bessere Praxis der Bundesstaaten seines US-amerikanischen Exils aus.

158 Dagegen ist wider die hohe Zustimmung nichts kritisch einzuwenden. Zwar ist es auch schon vorgekommen, daß eine vom Parlament einhellig beschlossene Verfassungsänderung beim Referendum vom Volk verworfen wurde - so geschehen in Hessen 1995, vgl. Jung, Volksentscheide (Fn. 9), S. 567-570 -, aber dies zeigte nur, daß die politische Klasse (jedenfalls bei diesem Thema) von der Basis allzu sehr ,abgehoben“ hatte, und die direktdemokratische Korrektur war dann sinnvoll. So aber war die Situation in Berlin bei dieser Reform erfreulicherweise nicht. 
stellt man aber, daß tatsächlich eine solche Differenz bestand - daß also die $\mathrm{Zu}$ stimmung zu der geplanten Reform im Verlauf jener fünf Wochen wuchs -, ist weitgehend offen, wie das geschehen sein soll. Das matte „Engagement“ der Parteien des Abgeordnetenhauses dürfte kaum ursächlich gewesen sein, und daß die Werbung des „Bündnisses für Direkte Demokratie“ bei aller Begeisterung der Aktiven so viel Erfolg hatte, muß aus den genannten quantitativen Gründen bezweifelt werden.

Inhaltlich dürfte sich die Ablehnung jener Reform im wohlhabenden bürgerlichen Westen aus zwei Strängen gespeist gaben: Zur altliberalen Auffassung, daß die Demokratie durch Repräsentation „,vergütet“ werde, gesellte sich wohl eine dumpfe Angst vor der „Begehrlichkeit der Massen“, die der Rechtsstaat womöglich nicht mehr zügeln könnte. Umgekehrt war die hohe Zustimmung in den östlichen und ärmeren Bezirken der Stadt wohl weniger der massenhaften Einsicht zu verdanken, wie notwendig es sei, die Volksgesetzgebung zu erleichtern; vielmehr dürfte eine naive Volks- und Demokratiegläubigkeit aus Restbeständen sozialistischen Denkens mitgespielt haben.

Die generelle Einschätzung des Abstimmungsergebnisses war freundlich: Damit bewiesen die Stimmberechtigten Berlins, „daß sie ihre demokratischen Rechte ernstnehmen“, kommentierte Efler, der Sprecher des Bündnisses. „Sie wollen sich in die Politik einmischen können.“159 „Berliner wollen mitbestimmen“, formulierte die „tageszeitung“ als Schlagzeile ${ }^{160}$, und die „Netzeitung“ konstatierte sachlich: „Berliner können künftig leichter aufbegehren.“'161

Aus prinzipieller Perspektive wäre zunächst zu betonen, daß mit dieser dritten Volksabstimmung innerhalb von elf Jahren die Bürgerinnen und Bürger Berlins direkte Demokratie weiter „üben“ konnten. Daß dies wiederum bei einem Referendum, also „von oben“ ausgelöst, geschah, war kein Schaden. Historisch gesehen, wurde ja in den alten demokratischen Republiken Schweiz und USA die direkte Demokratie auf diesem Wege eingeführt. Zuerst stimmten die Bürger über ihre Verfassungen ab, dann auch über Parlamentsgesetze, und erst später kam die Volksgesetzgebung hinzu. Bemerkenswerterweise schien sich zumindest das Berliner Verfassungsleben ähnlich zu entwickeln. „Üben“ hieß in diesem Falle, die direkte Demokratie von der Höhe einer abstrakten Idee auf die Ebene einer praktisch erfahrenen politischen Möglichkeit zu transponieren. Es war wichtig für die direkte Demokratie im Land Berlin, aber auch für die einschlägigen Diskussionen auf Bundesebene -, daß möglichst viele Bürgerinnen und Bürger einmal erlebt hatten, wie solch ein Verfahren abläuft und wie unaufgeregt und sicher - wie eine Wahl - eine Volksabstimmung praktiziert werden kann. Diesen

159 Berliner stimmen für mehr direkte Demokratie, in: Tsp. Nr. 19316 v. 19.9.2006.

160 taz Nr. 8078 v. 19.9.2006.

161 http://www.netzeitung.de/deutschland/landtagswahlen2006/441255.html (19.9.2006). 
wichtigen Erfahrungen, die „unten“ gemacht wurden, entsprach eine Selbstbindung derer da „oben“. Je mehr Volksabstimmungen praktisch durchgeführt wurden - ruhig und ohne Komplikationen -, um so weniger konnte weiter fundamentalistisch argumentiert werden: daß die Bürgerinnen und Bürger die komplizierten Sachverhalte gar nicht verstünden, daß sie sich von Demagogen aller Art verführen ließen, daß sie nur ,aus dem Bauch heraus“ entscheiden würden, und was der üblen Nachrede gegen die direkte Demokratie mehr war. 